\title{
Combined effects of tidal and rotational distortions on the equilibrium configuration of low-mass, pre-main sequence stars ${ }^{\star}$
}

\author{
N. R. Landin ${ }^{1}$, L. T. S. Mendes ${ }^{1,2}$, and L. P. R. Vaz ${ }^{1}$ \\ 1 Depto. de Física, Universidade Federal de Minas Gerais, CP 702, 31270-901 - Belo Horizonte, MG, Brazil \\ e-mail: [nlandin;lpv]@fisica.ufmg.br \\ 2 Depto. de Engenharia Eletrônica, Universidade Federal de Minas Gerais, CP 702, 31270-901 - Belo Horizonte, MG, Brazil \\ e-mail: luizt@cpdee.ufmg.br
}

Received 2 August 2007 / Accepted 26 October 2008

ABSTRACT

\begin{abstract}
Context. In close binary systems, the axial rotation and the mutual tidal forces of the component stars deform each other and destroy their spherical symmetry by means of the respective disturbing potentials.

Aims. We present new models for low-mass, pre-main sequence stars that include the combined distortion effects of tidal and rotational forces on the equilibrium configuration of stars. Using our theoretical results, we aim at investigating the effects of interaction between tides and rotation on the stellar structure and evolution.

Methods. The Kippenhahn \& Thomas (1970, in Stellar Rotation, ed. A. Slettebak) approximation, along with the Clairaut-Legendre expansion for the gravitational potential of a self-gravitating body, is used to take the effects of tidal and rotational distortions on the stellar configuration into account.

Results. We obtained values of internal structure constants for low-mass, pre-main sequence stars from stellar evolutionary models that consider the combined effects of rotation and tidal forces due to a companion star. We also derived a new expression for the rotational inertia of a tidally and rotationally distorted star. Our values corresponding to standard models (with no distortions) are compatible with those available in literature. Our distorted models were successfully used to analyze the eclipsing binary system EK Cep, reproducing the stellar radii, effective temperature ratio, lithium depletion, rotational velocities, and the apsidal motion rate in the age interval of $15.5-16.7 \mathrm{Myr}$.

Conclusions. In the low-mass range, the assumption that harmonics greater than $j=2$ can be neglected seems not to be fully justified, although it is widely used when analyzing the apsidal motion of binary systems. The non-standard evolutionary tracks are cooler than the standard ones, mainly for low-mass stars. Distorted models predict more mass-concentrated stars at the zero-age main-sequence than standard models.
\end{abstract}

Key words. stars: evolution - stars: interiors - stars: rotation - stars: pre-main sequence - stars: binaries: close stars: low-mass, brown dwarfs

\section{Introduction}

A binary system consists of two stars that rotate around their own axes and, at the same time, revolve around the center of mass of the system. Sometimes, the intrinsic rotation axis and the orbital one are aligned since the beginning of the formation process. Usually, the orbit begins with a considerable eccentricity and the component stars are not synchronized with the orbital angular velocity. However, due to the inertial forces that take place, the system tends to align its axes, to synchronize the rotational angular velocity of the components with the orbital angular velocity at the periastron passage and, finally, to circularize the orbit. Extensive spectroscopic evidence reveals that the components of close binary (in general, non-eccentric) systems do rotate with an angular velocity, $\Omega$, which is generally equal to the Keplerian angular velocity, $\omega_{\mathrm{K}}$, of the orbital motion around a common center of mass, so that

$\Omega \cong \omega_{\mathrm{K}}=\sqrt{G \frac{M_{1}+M_{2}}{R^{3}}}$.

* The full version of Table 1 is only available in electronic form at the CDS via anonymous ftp to cdsarc.u-strasbg.fr $(130.79 .128 .5)$ or via

http://cdsweb.u-strasbg.fr/cgi-bin/qcat?J/A+A/494/209
However, occasionally $\Omega$ is much larger than $\omega_{\mathrm{K}}$ - the sense of rotation being direct in every known case. In systems exhibiting circular orbits, synchronism between rotation and revolution may usually (though not always) be expected to exist, while components describing eccentric orbits, though in general rotating faster than their mean orbital angular velocity, can still be synchronized with the orbital motion at the periastron.

When both components are on the main sequence, the most massive one is also larger in radius and hotter, and is called primary, both in photometric (the star eclipsed in the deepest minimum of the light curve) and in spectroscopic studies. In other evolutionary stages, however, it is a common situation that the most massive component is not the larger or the one of higher effective temperature. It continues being designed as "primary" in spectroscopic studies, but referred to as "secondary" in light curve analysis, due to the lower effective temperature.

One important aspect of the evolution of close binaries is the dynamical evolution due to tidal interaction, which is reflected in the rotation of the stars and in the eccentricity of their orbits. Tidal deformation due to the companion would be symmetric about the line joining their centers, if there were no dissipation of kinetic energy into heat. It is this dissipation that induces a phase shift in the tidal bulge, and the tilted mass distribution, 
then, exerts a torque on the star, leading to an exchange of angular momentum between its spin and the orbital motion. Theory distinguishes two components in the tide, namely, equilibrium tide and dynamical tide (Zahn 1989):

- equilibrium tide is the hydrostatic adjustment of the structure of the star to the perturbing force exerted by the companion. The dissipation mechanism acting on this tide is the interaction between the convective motions and the tidal flow (Zahn 1966);

- dynamical tide is the dynamical response to the tidal force exerted by the companion; it takes into account the elastic properties of the star, and the possibilities of resonances with its free modes of oscillation. The dissipation mechanism acting on this tide is the departure from adiabaticity of the forced oscillation, due to the radiative damping (Zahn 1975).

To describe the tidal process in massive main-sequence stars, which have convective cores and radiative envelopes, it is necessary to use a theory that accounts for dynamical effects which arise due to tidal forces (Zahn 1977; Savonije \& Papaloizou 1983). Witte \& Savonije (1999a,b, 2001) published extensive studies of the role played by close resonances with eigenmodes of these early type stars during the orbital decay (see also Willems et al. 2003). On the other hand, for low-mass mainsequence stars and giants, which have extended convective envelopes, retardation of the equilibrium tide due to the viscosity of turbulent eddies in the envelope is usually assumed to be the cause of the tidal torque. Savonije \& Witte (2002) calculated the tidal interaction of a uniformly rotating $1 M_{\odot}$ star with a orbiting companion at various phases of evolution from the zero-age main-sequence (ZAMS) to core hydrogen exhaustion. Their results indicate that effects related to stellar rotation can considerably enhance the speed of tidal evolution in low-mass binary systems. In another paper, Witte \& Savonije (2002) showed that energy dissipation through resonant dynamic tides may dominate convective damping of equilibrium tides in solar-type stars.

In standard models, the stars are assumed to be spherically symmetric. However, the spherical symmetry will be destroyed if a disturbing potential exists, as it happens for rotating stars either isolated or in binary (multiple) systems, or due to tidal forces (gravitational influence of a companion), present in binary systems.

In the case of a rotating star in a binary system, both rotational and tidal forces distort its shape from the spherical symmetry. The analytic determination of these combined effects is complex and approximate methods have been used in the literature, with one of the distorting forces (generally rotation) being analyzed in approximate ways (Mohan et al. 1990). Chandrasekhar (1933) developed the theory of distorted polytropes and Kopal $(1972,1974)$ developed the concept of Roche equipotentials and coordinates to study the combined effects of tidal forces and rotation on stars. Kippenhahn \& Thomas (1970) (KT70) devised a method for introducing the effects of rotation in existing one-dimensional evolutionary codes that became widely adopted in the literature (see e.g. Endal \& Sofia 1976; Pinsonneault et al. 1990; Fliegener \& Langer 1995; Chaboyer et al. 1995; Meynet \& Maeder 1997; Mendes et al. 1999; Claret 1999). In the case of massive stars, the effects of rotation on their structure and evolution were studied by various authors (e.g. Meynet \& Maeder 1997, 2000; Maeder \& Zahn 1998; Heger et al. 2000; Maeder \& Meynet 2000, 2001), some of which present also grids of stellar models for rotating, massive stars.
In the low-mass range, Pinsonneault et al. (1990), Martín \& Claret (1996) and Mendes et al. (1999) presented exploratory studies on the influence of rotation on Li depletion in pre-main sequence (MS) evolution, and Landin et al. (2006) provided grids of non-gray rotating pre-MS models from $0.085 M_{\odot}$ to $3.8 M_{\odot}$.

Mohan et al. (1990) presented a method for calculating the equilibrium structure of a rotationally and tidally distorted primary component in a synchronously rotating binary system. Their method assumes a Roche model potential and uses the KT70 method for computing the corrections due to rotation and tides. Their results for stellar models of 10,5 , and $2.5 M_{\odot}$, for which the mass ratio $q$ of the secondary component to the primary component was set to 0.1 , indicate that rotational effects are more important than those produced by tidal distortions.

In this paper, we present a new version of the ATON code (Ventura et al. 1998) that treats the combined effects of tidal and rotational distortions on a star. This allows us, for example, to obtain the apsidal motion constants of the primary component of a binary system that rotates and suffers the tidal effects of the secondary component. Instead, however, of using a Roche-type potential, which considers both binary components as point masses, we adopt the more precise technique based on the Clairaut-Legendre expansion for the potential energy of a self-gravitating body (Kopal 1959). The paper is organized as follows: in Sect. 2 we give a brief account of theoretical apsidal motion calculations in the literature. Section 3 presents the KT70 method, proposed for determining the equilibrium structures of rotationally and tidally distorted stellar models, in which the non-spherical stellar equations can be easily obtained from the spherical ones. The technique for introducing the combined tidal and rotational effects is described in Sect. 4, in which we present, also, new calculations of internal structure constants extended to the pre-MS phase. The results are presented in the Sect. 5. Discussion and comparisons with observed apsidal motion rates are given in Sect. 6.

\section{Apsidal motion and internal structure constants}

The internal structure constants $k_{2}, k_{3}$ and $k_{4}$, also known as apsidal motion constants, are important in stellar astrophysics. They are mass concentration parameters that depend on the mass distribution throughout the star. There is, however, a direct relation between the gravitational field of a non-spherical body and the internal density concentration in that body (Sahade \& Wood 1978).

From a theoretical point of view, the values of $k_{j}(j=2,3$, 4) depend on the model used. For the Roche model, in which the whole stellar mass is concentrated at its center, the $k_{j}$ values are all equal to zero, while for a homogeneous model $k_{2}=3 / 4$, $k_{3}=3 / 8$ and $k_{4}=1 / 4$. The values of the internal structure constants are essential to compute the theoretical apsidal motion rates in close binaries, and the comparison with the observations constitutes an important test for evolutionary models. The most centrally concentrated stars have the lowest values of $k_{j}$ and the longest values of apsidal periods (Eq. (6)).

It can be shown that the theoretical apsidal rate $\dot{\omega}$, in radians per cycle, is given in terms of the internal structure constants by (Martynov 1973; Hejlesen 1987, hereafter H87)

$$
\frac{\dot{\omega}}{2 \pi}=\sum_{i=1}^{2} \sum_{j=2}^{4} c_{j i} k_{j i},
$$


where $i$ denotes the component star $(1=$ primary, $2=$ secondary $)$ and $j$ the harmonic order. Generally, terms of order higher than $j=2$ are very small so that only values of $k_{2 i}$ enter in Eq. (2).

The internal structure constants are important in other astrophysical aspects, since synchronization and circularization time scales in close binaries depend on $k_{2}$ (Zahn 1977). Other applications are in the computation of rotational angular momenta (Sect.4.4), where gyration radii (defined in Eq. (58)) can be expressed as a linear function of the apsidal motion constants (Ureche 1976), and in the determination of the effect of binarity in the geometry of the stellar surfaces due to rotation and tides (Ruciński 1969; Kopal 1978).

The first analytical expression for the apsidal motion period in close binaries in terms of stellar masses, relative radii and internal structure constants of the component stars was given by Russell (1928) and later improved by Cowling (1938). Chandrasekhar (1933) used polytropic models to predict internal structure constants for main-sequence stars. At that time, the large uncertainties of the observational data, as well as the use of polytropic models with an arbitrary index $n$, were responsible for the apparently good agreement between observed and predicted values of $\log k_{2}$.

By using more realistic stellar models, different authors derived more elaborated expressions for the apsidal motion period, separating rotational and tidal contributions to the total apsidal motion rate. The apsidal motion test was also applied to polytropic models by Sterne (1939), Brooker \& Olle (1955) and, later, to early theoretical stellar models at the ZAMS, by Schwarzschild (1958) and Kushawa (1957), both using the old Keller \& Meyerott (1955) opacities. At that time, the theoretical values of $k_{2}$ were systematically larger than those obtained by observations, which led to the interpretation that real stars were more centrally condensed than predicted by models. This discrepancy persisted during several decades; more recently, however, Claret \& Willems (2002) and Willems \& Claret (2003) have found no systematic effects in the sense that models are less mass concentrated than real stars, for binaries with accurately known absolute dimensions. Jeffery (1984) and H87 computed internal structure constants for stars within the main sequence. The former used Carson (1976) opacities, while the latter used opacity tables by Cox \& Stewart (1969).

In more recent years, the most extensive series of theoretical works in the literature regarding apsidal motion constants is that one from Claret and collaborators, some of which are briefly described here. Claret \& Giménez (1989a, hereafter CG89a) presented a detailed grid of evolutionary stellar models during the hydrogen burning phases, including apsidal motion constants. Claret \& Giménez (1991) studied the effect of the core overshooting and mass loss on the internal density concentration of main-sequence stars. With more updated input physics, Claret \& Giménez (1992, hereafter CG92) computed stellar models together with internal together with internal structure constants. Those models showed a general tendency to be cooler and more centrally concentrated in mass than their previous computations for the typical masses where apsidal motion is observed. Claret \& Giménez $(1992,1993)$ and Claret (1995) were able to reduce the discrepancies between theoretical and observed values of $k_{2}$ at acceptable levels for systems whose relativistic contributions were small. Claret (1999) took into account the effect of rotation on the internal structure of stars and found that it strongly depends on the distortion of the configuration. Claret $\&$ Willems (2002) revised the status of the apsidal motion test to stellar structure and evolution. They increased the observational sample by about $50 \%$ in comparison with previous works and took into account the effects of dynamic tides to determine the contribution of the tidal distortion to the predicted apsidal motion rate; they found a good agreement between observed and theoretical apsidal motion rates. The most recent internal structure constants for main-sequence stars are those contained in Claret (2004, 2005, 2006b, 2007, hereafter C04, C05, C06b and $\mathrm{C} 07$, respectively). They were calculated with new stellar models based on updated physics computed with different metallicities: $(X, Z)=(0.70,0.02)[C 04] ;(X, Z)=(0.754,0.002)$ and $(0.748,0.004)[\mathrm{C} 05] ;(X, Z)=(0.730,0.010)$ and $(0.739,0.007)$ $[\mathrm{C} 06 \mathrm{~b}] ;$ and $(X, Z)=(0.64,0.04),(0.58,0.06)$ and $(0.46,0.10)$ [C07].

\subsection{Perturbations and the apsidal motion}

The longitude of periastron of a binary orbit, $\omega$, defines the direction of the line of apsides in the orbital plane. It is constant, in the orbit of a system consisting of two gravitating bodies, only if all the three following conditions are valid: (i) the bodies can be regarded as point masses; (ii) they move in accordance with Newton law of gravitation $\left(r^{-2}\right)$; and (iii) the two bodies form a gravitationally isolated system. However, if any of these conditions fails, the size, form, and spatial position of the orbit will vary. The most readily detectable effect is a variation in the value of $\omega$ with time that is referred to as rotation (advance or recession) of the line of apsides. For a more detailed discussion of this subject, see, for instance, the works of Batten (1973) or Claret \& Giménez (2001).

Several types of perturbations exist and can lead to rotation of apsides, such as mutual tidal distortion of the components, distortion of the components due to axial rotation, relativistic effects, presence of a third body, and recession due to a resisting circumbinary medium. The axial rotation and the mutual tidal forces of close binary systems' components will deform each other and destroy their spherical symmetry, by means of the respective disturbing potentials. Besides the changes in the stellar structure, described in Sect. 4, these disturbing potentials produce an observed change in $\omega$ which is the sum of the effects caused by each component (Batten 1973). The total rate of apsidal advance per orbital revolution, $\dot{\omega}$, is

$\frac{\dot{\omega}}{2 \pi}=\frac{P}{U}=k_{21} c_{21}+k_{22} c_{22}$,

where $P$ is the anomalistic orbital period, $U$ is the apsidal motion period, and

$c_{2 i}=\left[\left(\frac{\Omega_{i}}{\omega_{\mathrm{K}}}\right)^{2}\left(1+\frac{M_{3-i}}{M_{i}}\right) f(e)+\frac{15 M_{3-i}}{M_{i}} g(e)\right]\left(\frac{R_{i}}{A}\right)^{5}$,

where $i=1,2$ stands for the primary and the secondary stars, respectively; $M_{i}$ and $R_{i}$ are stellar mass and radius of component $i$; $A$ is the semi-major axis; $e$ is the orbital eccentricity; and the functions $f(e)$ and $g(e)$ are defined as

$f(e)=\left(1-e^{2}\right)^{-2}$ and $g(e)=\frac{\left(8+12 e^{2}+e^{4}\right) f(e)^{2.5}}{8}$,

$\left(\Omega_{i} / \omega_{\mathrm{K}}\right)$ being the ratio between the actual angular rotational velocity of the stars and that corresponding to synchronization with the average orbital velocity. Equation (3) is a special case of Eq. (2), in which only the second order harmonics are taken into account. The first term in Eq. (4) represents the contribution to the total apsidal motion given by rotational distortions and the second term corresponds to the tidal contributions. 
With the exception of the $k_{2 i}$, all parameters in Eq. (3) can be determined from photometric and spectroscopic analysis. The empirical weighted average of the internal structure constants for individual systems can be given by

$\bar{k}_{2 \mathrm{obs}}=\frac{1}{c_{21}+c_{22}} \frac{P}{U}=\frac{1}{c_{21}+c_{22}} \frac{\dot{\omega}}{2 \pi}$.

Equations (4) and (6) show that $\bar{k}_{2 \text { obs }}$ depend on our knowledge of the rotation velocities of the component stars. In most binaries with good absolute dimensions, the rotation velocities of the individual components are known through spectroscopic analysis. Since the average orbital rotation, or Keplerian velocity, is a function of the orbital period, the ratio of rotational velocities in Eq. (4), namely $\Omega_{i} / \omega_{\mathrm{K}}$, is well determined in these binaries (Claret \& Giménez 1993). For systems without observational determinations, the best approach is to assume that the components are synchronized with the orbital velocity at periastron, where the tidal forces are at maximum. The rotation velocities are related by (Kopal 1978)

$\omega_{\mathrm{P}}^{2}=\frac{(1+e)}{(1-e)^{3}} \omega_{\mathrm{K}}^{2}$

where $\omega_{\mathrm{P}}$ is the angular velocity at periastron, $e$ is the orbital eccentricity, and $\omega_{\mathrm{K}}$ is the Keplerian angular velocity (Eq. (1)). Claret \& Giménez (1993) checked the validity of this approximation, achieving a good agreement between the observed and the predicted rotational velocities by assuming synchronization at periastron (see their Fig. 6).

The mean $k_{2 i}$ values, obtained through Eq. (6), can be compared with those derived from theoretical models (Eq. (10)). However, the observed mean values of $k_{2}$ obs should be first corrected from non-distortional effects, like relativistic, third body, and interstellar medium contributions.

\subsection{Internal structure constants for spherically symmetric configurations}

Internal structure constants $\left(k_{j}\right)$ can be approximately computed based on the simple (and unrealistic) assumption that stars can be described by spherically symmetric models. These approximate $k_{j}$ have been computed for comparison with observed rates of apsidal motion.

The Radau's differential equation (Kopal 1959) is numerically integrated throughout all the structure with a 4th-order Runge-Kutta method (Press et al. 1992):

$r \frac{\mathrm{d} \eta_{j}}{\mathrm{~d} r}+6 \frac{\rho(r)}{\bar{\rho}(r)}\left(\eta_{j}+1\right)+\eta_{j}\left(\eta_{j}-1\right)=j(j+1)$,

where $\eta_{j}(0)=j-2(j=2,3,4), \rho(r)$ is the local density at a distance $r$ from the center, and $\bar{\rho}(r)$ is the mean density within the inner sphere of radius $r$. The resulting value of the function $\eta_{j}(R)$, which satisfies Radau's equation with $R$ being the radius of the configuration, is used to obtain the individual values of $k_{j}$,

$k_{j}=\frac{j+1-\eta_{j}(R)}{2\left(j+\eta_{j}(R)\right)}$.

Because the observed motion of apsides is the sum of the motion produced by both stars, the quantities $k_{2 i}$ ( $i=1,2$ for the primary and the secondary star, respectively) cannot be observationally determined separately. So, the theoretical counterpart of Eq. (6) is the weighted mean value of the individual theoretical apsidal motion constant,

$\bar{k}_{2, \text { theo }}=\frac{c_{21} k_{21, \text { theo }}+c_{22} k_{22, \text { theo }}}{c_{21}+c_{22}}$,

where $c_{21}$ and $c_{22}$ are computed through Eq. (4) by using absolute dimensions, and $k_{21 \text {,theo }}$ and $k_{22 \text {,theo }}$ are the theoretical apsidal motion constants for the primary and the secondary, respectively, obtained from the stellar models (Eq. (9)) for the corresponding mass and radius of each component. Before performing the apsidal motion comparison, one has to check if the models are able to reproduce basic stellar parameters, such as effective temperatures, and to predict a common age for the two components. A good discussion of this subject is given by Claret \& Giménez (1993). In Sect. 7 we show our predictions about apsidal motion for a chosen binary, comparing them with observational data.

\section{The Kippenhahn and Thomas formulation}

The KT70 method is a strategy for considering disturbing potentials in evolutionary stellar models more realistically, so that the distortion produced by a given disturbing potential is entirely included in the total potential function.

To clarify how these disturbing effects were taken into account in the KT70 method, the equations are re-derived here. In this formulation, the spherically symmetric surfaces, normally used in standard stellar models, are replaced by suitable nonspherical equipotential surfaces characterized by the total potential $\psi$, the mass $M_{\psi}$ enclosed by the corresponding equipotential surface whose surface area is $S_{\psi}$ and encloses a volume $V_{\psi}$, and $r_{\psi}$, the radius of the topologically equivalent sphere with the same volume $V_{\psi}$, enclosed by the equipotential surface.

For any quantity $f$ varying over an equipotential surface, we can define its mean value as

$\langle f\rangle=\frac{1}{S_{\psi}} \int_{\psi_{\text {const. }}} f \mathrm{~d} \sigma$

where $S_{\psi}=\int_{\psi_{\text {const. }}} \mathrm{d} \sigma$, and $\mathrm{d} \sigma$ is the surface element.

The local effective gravity is given by $g=\frac{\mathrm{d} \psi}{\mathrm{d} n}$, where $\mathrm{d} n$ is the (non-constant) separation between two successive equipotentials $\psi$ and $\psi+\mathrm{d} \psi$, so that we have

$\langle g\rangle=\frac{1}{S_{\psi}} \int_{\psi_{\text {const. }}} \frac{\mathrm{d} \psi}{\mathrm{d} n} \mathrm{~d} \sigma$,

$\left\langle g^{-1}\right\rangle=\frac{1}{S_{\psi}} \int_{\psi_{\text {const. }}}\left(\frac{\mathrm{d} \psi}{\mathrm{d} n}\right)^{-1} \mathrm{~d} \sigma$

The volume between the surfaces $\psi$ and $\psi+\mathrm{d} \psi$ is given by

$\mathrm{d} V_{\psi}=\int_{\psi_{\text {const. }}} \mathrm{d} n \mathrm{~d} \sigma=\mathrm{d} \psi \iint_{\psi_{\text {const. }}}\left(\frac{\mathrm{d} n}{\mathrm{~d} \psi}\right) \mathrm{d} \sigma=\mathrm{d} \psi S_{\psi}\left\langle g^{-1}\right\rangle$,

from which we obtain

$\mathrm{d} \psi=\frac{1}{S_{\psi}\left\langle g^{-1}\right\rangle} \mathrm{d} V_{\psi}=\frac{1}{S_{\psi}\left\langle g^{-1}\right\rangle} \frac{\mathrm{d} M_{\psi}}{\rho(\psi)}$,

and the volume of the topologically equivalent sphere is given by $V_{\psi}=\frac{4 \pi}{3} r_{\psi}^{3}$. 
Equation (15) can be combined with the general form of the hydrostatic equilibrium equation,

$\frac{\mathrm{d} P}{\mathrm{~d} \psi}=-\rho, \quad$ to give

$\frac{\mathrm{d} P}{\mathrm{~d} M_{\psi}}=-\frac{G M_{\psi}}{4 \pi r_{\psi}^{4}} f_{\mathrm{p}}$,

where $f_{\mathrm{p}}$ is given by

$f_{\mathrm{p}}=\frac{4 \pi r_{\psi}^{4}}{G M_{\psi}} \frac{1}{S_{\psi}\left\langle g^{-1}\right\rangle}$.

With these corrections, the four stellar structure equations, with $M_{\psi}$ as the independent variable, become

$\frac{\mathrm{d} P}{\mathrm{~d} M_{\psi}}=-\frac{G M_{\psi}}{4 \pi r_{\psi}^{4}} f_{\mathrm{p}}$,

$\frac{\mathrm{d} r_{\psi}}{\mathrm{d} M_{\psi}}=\frac{1}{4 \pi r_{\psi}^{2} \rho}$,

$\frac{\mathrm{d} L_{\psi}}{\mathrm{d} M_{\psi}}=\epsilon-T \frac{\partial S}{\partial t}$

$\frac{\mathrm{d} T_{\psi}}{\mathrm{d} M_{\psi}}=-\frac{G M_{\psi} T}{4 \pi r_{\psi}^{4} P} \nabla, \quad \nabla=\left\{\nabla_{\text {rad }}, \frac{f_{\mathrm{t}}}{f_{\mathrm{p}}} \nabla_{\text {conv }}\right\}$,

where $f_{\mathrm{p}}$ is given by Eq. (18) and

$f_{\mathrm{t}}=\left(\frac{4 \pi r_{\psi}^{2}}{S_{\psi}}\right)^{2} \frac{1}{\langle g\rangle\left\langle g^{-1}\right\rangle}$.

In the case of isolated and non-rotating stars, $f_{\mathrm{p}}=f_{\mathrm{t}}=1$, and the original stellar structure equations are recovered. In order to obtain the internal structure of a distorted gas sphere, the set of Eqs. (19) must be numerically integrated under suitable boundary conditions.

This formulation was largely used in the literature mainly due to the easiness of its implementation in existing evolutionary codes (e.g. Endal \& Sofia 1976; Law 1980; Pinsonneault 1988; Martín \& Claret 1996; Mendes et al. 1999).

\section{Tidal and/or rotational distortions on the equilibrium structure of stars}

We consider the effects of tidal forces, as well as the combined effects of tidal forces and rotation, on the stellar tructure and implemented such effects in the ATON code. Mendes (1999) had already introduced the effects of rotation alone in it. The tidal and rotational effects in stellar models were implemented according to the treatment derived by KT70 and modified by Endal \& Sofia (1976), who in turn used a more refined function to take into account terms related to the distortion of the stars, namely the Clairaut-Legendre expansion for the gravitational potential of a self-gravitating body (Kopal 1959). The calculations are done within the framework of static tides; in the case of dynamic tides, a more refined treatment is required (see Claret \& Willems 2002; Willems \& Claret 2003).

In our model, tides and axial rotation are the two physical causes of the stellar configuration deviating from a spherical form. The centrifugal pseudo-potential terms arising from the orbital motion around the center of mass of the system do contribute, also, for the departure from sphericity, but we do not consider these terms in the present work, yet. These terms will be included in future works. We treat three different situations: 1) rotation acting alone; 2) tidal forces acting alone; and 3) a combination of both effects distorting the star. The special case of the evolution of one of the components in a binary system is considered, assuming that (i) it rotates about an axis perpendicular to the orbital plane; (ii) from the orbital point of view, the system is considered to be at rest, i.e., the stars do not revolve around each other; and, particularly in this work; (iii) the ratio of the mutual separation to the radius of the evolving (distorted) star is assumed to be constant. The value of this ratio is an input parameter that depends on the system to be reproduced; in close binaries, the separation of the stars is often less than 10 times their radii (Hilditch 2001). The equipotential surfaces can be written as an expansion of the tesseral harmonics ${ }^{1} Y_{j}^{i}$,

$r\left(r_{0}, \theta, \phi\right)=r_{0}\left[1+\sum_{i, j} Y_{j}^{i}\left(r_{0}, \theta, \phi\right)\right]$,

where $r_{0}$ is the mean radius of the corresponding equipotential surface. The tesseral harmonics can be written as

$Y_{j}^{i}\left(r_{0}, \theta, \phi\right)=K\left(r_{0}\right) P_{j}^{i}(\theta, \phi)$,

where $K\left(r_{0}\right)$ is the radial part of the tesseral harmonics and its angular part, $P_{j}^{i}$, being functions associated with Legendre polynomials.

\subsection{Rotational distortion}

Rotation alone would render the star a rotational spheroid flattened at the poles. In what follows we are considering conservative rotation, which means that the centrifugal acceleration can be derived from a potential,

$\Omega^{2} s \mathrm{e}_{\mathrm{s}}=-\nabla V_{\mathrm{rot}}$,

where $s=r \sin \theta$ is the perpendicular distance to the rotation axis of the star. A necessary and sufficient condition for the conservative case is that $\Omega=\Omega(s)$ (Kippenhahn \& Weigert 1994), which means rotation is constant on cylinders. Rigid body rotation is obviously a special case of conservative rotation.

Following Kopal (1959) and later Endal \& Sofia (1976), the total potential is divided in three parts according to Eqs. (24), where $\psi_{\mathrm{s}}$ is the spherically symmetric part of the gravitational potential, $\psi_{\mathrm{r}}$ is the cylindrically symmetric potential due to rotation, and $\psi_{\mathrm{d}}^{(\mathrm{rot})}$ is the cylindrically symmetric part of the gravitational potential due to the distortion of the figure of the star caused by rotation. If the coordinates of the point $P$ are the radius $r$ and the polar angle $\theta$ (measured from the rotational axis), the components of the potential at $P$ can be written as

$\psi_{\mathrm{s}}=\frac{G M_{\psi}}{r}$

$\psi_{\mathrm{r}}=\frac{1}{2} \Omega^{2} \sin ^{2} \theta$

$\psi_{\mathrm{d}}^{(\mathrm{rot})}=\sum_{j=2}^{\infty} \frac{4 \pi G}{(2 j+1) r^{j+1}} \int_{0}^{r_{0}} \rho \frac{\partial}{\partial r_{0}^{\prime}}\left(r_{0}^{\prime j+3} Y_{j}^{i}\right) \mathrm{d} r_{0}^{\prime}$.

1 Tesseral harmonics are spherical harmonics of the form $\cos (m \phi) P_{l}^{m}(\cos \theta)$ and $\sin (m \phi) P_{l}^{m}(\cos \theta)$, for $m \neq l$. These harmonics are so named because the curves on which they vanish are $l-m$ parallels of latitude and $2 m$ meridians, which divide the surface of a sphere into quadrangles whose angles are right angles. 
In Eqs. (24), $M_{\psi}$ and $\Omega$ are respectively the mass and the angular velocity of the rotating star, while $r_{0}$, by virtue of the cylindrically symmetric rotational potential $\psi_{\mathrm{r}}$, corresponds to the radius of the equipotential surface (as given by Eq. (21)) at the angle $\theta_{0}$ defined such that $P_{2}\left(\cos \theta_{0}\right)=0, P_{2}$ being the secondorder Legendre polynomial. Here, the shape of rotating configurations is described according to the expansion given in Eq. (21), with $Y_{j}^{i}$ given by

$Y_{j}^{i}\left(r_{0}\right)=c_{i, j} \frac{2 j+1}{j+\eta_{j}\left(r_{0}\right)} \frac{r_{0}^{j+1}}{G M_{\psi}} P_{j}^{i}(\theta, \phi)$.

By limiting ourselves to first-order theory, the disturbing potential associated to rotation (Eq. (24b)) is such that it will invoke a single non-zero $Y_{j}^{i}$ term in Eq. (21), namely that one corresponding to $i=0, j=2$ (Kopal 1959, 1960):

$c_{0,2}=-\frac{1}{3} \Omega^{2}$.

So, in the case of rotational distortions acting alone, the equipotential surface now becomes

$r\left(r_{0}, \theta\right)=r_{0}\left[1+Y_{\text {rot }}\right]$.

in which we replaced $Y_{2}$ by $Y_{\text {rot }}$ for better clarity, and

$Y_{\text {rot }}=-\frac{\Omega^{2} r_{0}^{3}}{3 G M_{\psi}} \frac{5}{2+\eta_{2}\left(r_{0}\right)} P_{2}(\cos \theta)$,

where we have abbreviated, as it is customary,

$\eta_{2}=\frac{r_{0}}{Y_{\text {rot }}} \frac{\partial Y_{\text {rot }}}{\partial r_{0}}$

In short, $Y_{\text {rot }}$ is a measure of the deviation from sphericity caused by rotation. The quantity $\eta_{2}$ is of particular interest to our study, because the theoretical apsidal motion constant $k_{2}$ can be derived from it (see Sect. 2). The evaluation of $\eta_{2}$ can be done by numerically integrating the Radau's equation:

$r_{0} \frac{\mathrm{d} \eta_{2}}{d r_{0}}+6 \frac{\rho\left(r_{0}\right)}{\bar{\rho}\left(r_{0}\right)}\left(\eta_{2}+1\right)+\eta_{2}\left(\eta_{2}-1\right)=6$.

This equation is slightly different from Eq. (8). Here we use $j=$ 2 , and the spherical radius $r$ was replaced by $r_{0}$, the mean radius of the distorted configuration.

By virtue of the definition of $Y_{\text {rot }} \equiv Y_{2}$ as given by Eq. (28), the expression for $\psi_{\mathrm{d}}^{(\mathrm{rot})}$ simplifies to

$\psi_{\mathrm{d}}^{(\mathrm{rot})}=-\frac{4 \pi}{3 r^{3}} P_{2}(\cos \theta) \int_{0}^{r_{0}} \rho \frac{r_{0}^{\prime 7}}{M_{\psi}} \Omega^{2} \frac{5+\eta_{2}}{2+\eta_{2}} \mathrm{~d} r_{0}^{\prime}$

so that the total potential can be written as

$$
\begin{aligned}
\psi= & \psi_{\mathrm{s}}+\psi_{\mathrm{r}}+\psi_{\mathrm{d}}^{(\mathrm{rot})} \\
= & \frac{G M_{\psi}}{r}+\frac{1}{2} \Omega^{2} \sin ^{2} \theta \\
& -\frac{4 \pi}{3 r^{3}} P_{2}(\cos \theta) \int_{0}^{r_{0}} \rho \frac{r_{0}^{\prime 7}}{M_{\psi}} \Omega^{2} \frac{5+\eta_{2}}{2+\eta_{2}} \mathrm{~d} r_{0}^{\prime} .
\end{aligned}
$$

By defining the radial part of the axisymmetric tesseral harmonic $Y_{\text {rot }}$ as

$A\left(r_{0}\right)=\frac{\Omega^{2} r_{0}^{3}}{3 G M_{\psi}} \frac{5}{2+\eta_{2}}$, the radius of the equipotential surface, Eq. (27), can be rewritten as

$r\left(r_{0}, \theta\right)=r_{0}\left[1-A\left(r_{0}\right) P_{2}(\cos \theta)\right]$.

To relate $r_{0}$ to $r_{\psi}$, we evaluate the volume integral from $r=0$ to $r\left(r_{0}, \theta\right)$ to obtain

$V_{\psi}=\frac{4 \pi r_{0}^{3}}{3}\left[1+\frac{3}{5} A^{2}-\frac{2}{35} A^{3}\right]$.

For simplicity, the arguments of the term $A\left(r_{0}\right)$ were suppressed. From the previous equation, $r_{\psi}$ is then obtained as

$r_{\psi}=r_{0}\left[1+\frac{3}{5} A^{2}-\frac{2}{35} A^{3}\right]^{1 / 3}$.

Usually, $r_{\psi}$ is known and so $r_{0}$ can be calculated through Eq. (36) by means of an iterative procedure.

Since the local effective gravity is given by

$g=\frac{\partial \psi}{\partial n}=\left[\left(\frac{\partial \psi}{\partial r}\right)^{2}+\left(\frac{1}{r} \frac{\partial \psi}{\partial \theta}\right)^{2}\right]^{1 / 2}$

$g$ can be found by differentiation of Eq. (32). The integral in Eq. (32) and their derivatives must be evaluated numerically. Once the values of $\langle g\rangle$ and $\left\langle g^{-1}\right\rangle$ are known for a set of points on an equipotential surface, $S_{\psi}\langle g\rangle$ and $S_{\psi}\left\langle g^{-1}\right\rangle$ can be found, respectively, from Eqs. (12) and (13) by numerically integrating over $\theta$.

\subsection{Tidal distortion}

Tidal distortion acting alone would tend to elongate the star in the direction of the other component. Similarly to Eqs. (24), the total potential is divided in three parts $\psi_{\mathrm{s}}, \psi_{\mathrm{t}}$, and $\psi_{\mathrm{d}}^{\text {(tid) }}$, where $\psi_{\mathrm{s}}$ is again the spherically symmetric part of the gravitational, potential, $\psi_{\mathrm{t}}$ is the non-symmetric potential due to tidal forces, and $\psi_{\mathrm{d}}^{\text {(tid) }}$ is the non-symmetric part of the gravitational potential due to distortion of the star caused by the presence of the companion. If the coordinates of the point $P$ are the radius $r$, the polar angle $\theta$, and the azimuthal angle $\phi$, the components of the potential at $P$ can be written as:

$$
\begin{aligned}
\psi_{\mathrm{s}} & =\frac{G M_{\psi}}{r}, \\
\psi_{\mathrm{t}} & =\frac{G M_{2}}{R}\left[\sum_{j=2}^{\infty}\left(\frac{r_{0}}{R}\right)^{j} P_{j}(\lambda)\right], \\
\psi_{\mathrm{d}}^{(\mathrm{tid})} & =\sum_{j=2}^{\infty} \frac{4 \pi G}{(2 j+1) r^{j+1}} \int_{0}^{r_{0}} \rho \frac{\partial}{\partial r_{0}^{\prime}}\left(r_{0}^{\prime j+3} Y_{j}^{i}\right) \mathrm{d} r_{0}^{\prime} .
\end{aligned}
$$

In Eqs. (38), $M_{\psi}$ and $M_{2}$ are respectively the masses of the primary and the disturbing star; $R$ is the mutual separation between the centers of mass of the two stars; $\lambda=\cos \phi \sin \theta ; r_{0}$ is the radius of the equipotential surface at the angles $\left(\theta_{0}, \phi_{0}\right)$, defined such that

$B\left(r_{0}\right) P_{2}\left(\lambda_{0}\right)+C\left(r_{0}\right) P_{3}\left(\lambda_{0}\right)+D\left(r_{0}\right) P_{4}\left(\lambda_{0}\right)=0$,

where $\lambda_{0}=\cos \phi_{0} \sin \theta_{0}$ and the terms $B\left(r_{0}\right), C\left(r_{0}\right)$ and $D\left(r_{0}\right)$ are defined in Eqs. (47); and $P_{j}$ are the $j$ th-order Legendre polynomials. 
By virtue of the disturbing tidal potential given by Eq. (38b), and again restricting ourselves to first-order quantities only, it follows that the only non-zero $Y_{j}$ terms in Eq. (21) consistent with the equilibrium theory of tides are those for which $i=0, j=$ 2,3, 4 (the reader is referred to the works of Kopal 1959, 1960, for full details):

$c_{0, j}=G \frac{M_{2}}{R^{j+1}} \quad(j=2,3,4)$,

from which we have

$Y_{j}=\frac{M_{2}}{M_{\psi}} \frac{2 j+1}{j+\eta_{j}\left(r_{0}\right)}\left(\frac{r_{0}}{R}\right)^{j+1} P_{j}(\lambda), \quad(j=2,3,4)$,

where, as before,

$\eta_{j}=\frac{r_{0}}{Y_{j}} \frac{\partial Y_{j}}{\partial r_{0}}$

the equipotential surfaces are then described by

$r\left(r_{0}, \theta, \phi\right)=r_{0}\left[1+\sum_{j=2}^{4} Y_{j}\right]$

So, for tidal forces acting alone, the $Y_{j}$ are a measure of the deviation from sphericity due to those forces.

As in Sect. 4.1, the apsidal motion constants can be derived from $\eta_{j}$ by using our theoretical stellar models (see Sect. 2). The evaluation of the quantity above can be done by, again, numerically integrating the Radau's equation

$r_{0} \frac{\mathrm{d} \eta_{j}}{\mathrm{~d} r_{0}}+6 \frac{\rho\left(r_{0}\right)}{\bar{\rho}\left(r_{0}\right)}\left(\eta_{j}+1\right)+\eta_{j}\left(\eta_{j}-1\right)=j(j+1)$,

for each $j=2,3,4$.

Substituting $Y_{j}$ as given by Eqs. (41) in (38c) and carrying on the partial derivative in the latter, we get

$\psi_{\mathrm{d}}^{(\mathrm{tid})}=4 \pi G M_{2} \sum_{j=2}^{4} \frac{P_{j}(\lambda)}{(r R)^{j+1}} \int_{0}^{r_{0}} \rho \frac{r_{0}^{\prime 2 j+3}}{M_{\psi}} \frac{j+3+\eta_{j}}{j+\eta_{j}} \mathrm{~d} r_{0}^{\prime}$.

The total potential is then

$$
\begin{aligned}
\psi= & \psi_{\mathrm{s}}+\psi_{\mathrm{t}}+\psi_{\mathrm{r}}^{(\mathrm{tid})} \\
= & \frac{G M_{\psi}}{r}+\frac{G M_{2}}{R}\left[\sum_{j=2}^{\infty}\left(\frac{r_{0}}{R}\right)^{j} P_{j}(\lambda)\right] \\
& +4 \pi G M_{2} \sum_{j=2}^{4} \frac{P_{j}(\lambda)}{(r R)^{j+1}} \int_{0}^{r_{0}} \rho \frac{r^{\prime 2 j+3}}{M_{\psi}} \frac{j+3+\eta_{j}}{j+\eta_{j}} \mathrm{~d} r_{0}^{\prime} .
\end{aligned}
$$

By defining the radial parts of the non-symmetric tesseral harmonics $Y_{j}$ in Eq. (41) as

$$
\begin{aligned}
& B\left(r_{0}\right)=\frac{M_{2}}{M_{\psi}}\left(\frac{r_{0}}{R}\right)^{3} \frac{5}{2+\eta_{2}}, \\
& C\left(r_{0}\right)=\frac{M_{2}}{M_{\psi}}\left(\frac{r_{0}}{R}\right)^{4} \frac{7}{3+\eta_{3}}, \\
& D\left(r_{0}\right)=\frac{M_{2}}{M_{\psi}}\left(\frac{r_{0}}{R}\right)^{5} \frac{9}{4+\eta_{4}},
\end{aligned}
$$

we can describe the equipotential surfaces, Eq. (43), as

$r\left(r_{0}, \theta, \phi\right)=$

$$
r_{0}\left[1+B\left(r_{0}\right) P_{2}(\lambda)+C\left(r_{0}\right) P_{3}(\lambda)+D\left(r_{0}\right) P_{4}(\lambda)\right] .
$$

We evaluate the volume integral from $r=0$ to $r\left(r_{0}, \theta, \phi\right)$ given by Eq. (48) and obtain

$$
\begin{array}{r}
V_{\psi}=\frac{4 \pi r_{0}^{3}}{3}\left[1+\frac{3 B^{2}}{5}+\frac{3 C^{2}}{7}+\frac{2 B^{3}}{35}+\frac{6 B^{2} D}{35}+\frac{D^{2}}{3}\right. \\
\left.+\frac{4 B C^{2}}{35}+\frac{20 B D^{2}}{231}+\frac{6 C^{2} D}{77}+\frac{18 D^{3}}{1001}\right],
\end{array}
$$

where the arguments of the terms $B\left(r_{0}\right), C\left(r_{0}\right)$ and $D\left(r_{0}\right)$ were omitted. From Eq. (49), $r_{0}$ and $r_{\psi}$ are related by

$$
\begin{aligned}
r_{\psi}= & r_{0}\left[1+\frac{3 B^{2}}{5}+\frac{3 C^{2}}{7}+\frac{2 B^{3}}{35}+\frac{6 B^{2} D}{35}+\frac{4 B C^{2}}{35}\right. \\
& \left.+\frac{20 B D^{2}}{231}+\frac{6 C^{2} D}{77}+\frac{18 D^{3}}{1001}+\frac{D^{2}}{3}\right]^{\frac{1}{3}} .
\end{aligned}
$$

Similarly to the case of pure rotation, $r_{0}$ can be calculated from the value of $r_{\psi}$ through Eq. (50), by means of an iterative procedure.

The local effective gravity is now given by

$g=\frac{\partial \psi}{\partial n}=\left[\left(\frac{\partial \psi}{\partial r}\right)^{2}+\left(\frac{1}{r} \frac{\partial \psi}{\partial \theta}\right)^{2}+\left(\frac{1}{r \sin \theta} \frac{\partial \psi}{\partial \phi}\right)^{2}\right]^{\frac{1}{2}}$,

and it can be obtained by differentiation of Eq. (46). As before, integrals and derivatives must be evaluated numerically.

Finally, by integrating over $\theta$ and $\phi$, we find $S_{\psi}\langle g\rangle$ and $S_{\psi}\left\langle g^{-1}\right\rangle$ from Eqs. (12) and (13), respectively, once $\langle g\rangle$ and $\left\langle g^{-1}\right\rangle$ are known for a set of points on an equipotential surface.

\subsection{Combined effects of rotation and tides}

According to Kopal $(1960,1974)$, the combined effects of rotation and tides give rise to a total disturbing potential that can be expressed as the sum of the distortion terms corresponding to pure rotation or tides and of a number of interaction terms. The latter, however, are in general second-order terms; so, in the present work, we neglect those interaction terms since we are dealing with first-order theory only.

The total potential for the combined case is then composed by four parts, which represent the joint contributions from first-order rotational and tidal effects as respectively given by Eqs. (24) and (38): $\psi_{\mathrm{s}}$, the spherically symmetric part of the gravitational potential; $\psi_{\mathrm{r}}$, the cylindrically symmetric potential due to rotation; $\psi_{\mathrm{t}}$, the non-symmetric potential due to tidal forces; and $\psi_{\mathrm{d}} \equiv \psi_{\mathrm{d}}^{\text {(rot) }}+\psi_{\mathrm{d}}^{\text {(tid) }}$, the non-symmetric part of the gravitational potential due the to distortion of the star considering both effects. Again, we do not take into account the pseudopotential centrifugal terms due to the orbital motion in this approximation. So, the total potential for the combined case (to first-order approximation) at $P(r, \theta, \phi)$ is

$$
\begin{aligned}
\psi= & \psi_{\mathrm{s}}+\psi_{\mathrm{r}}+\psi_{\mathrm{t}}+\psi_{\mathrm{d}} \\
= & \frac{G M_{\psi}}{r}+\frac{1}{2} \Omega^{2} \sin ^{2} \theta+\frac{G M_{2}}{R}\left[1+\sum_{j=2}^{4}\left(\frac{r_{0}}{R}\right)^{j} P_{j}(\lambda)\right] \\
& -\frac{4 \pi}{3 r^{3}} P_{2}(\cos \theta) \int_{0}^{r_{0}} \rho \frac{r_{0}^{\prime 7}}{M_{\psi}} \Omega^{2} \frac{5+\eta_{2}}{2+\eta_{2}} \mathrm{~d} r_{0}^{\prime} \\
& +4 \pi G M_{2} \sum_{j=2}^{4} \frac{P_{j}(\lambda)}{(r R)^{j+1}} \int_{0}^{r_{0}} \rho \frac{r_{0}^{\prime 2 j+3}}{M_{\psi}} \frac{j+3+\eta_{j}}{j+\eta_{j}} \mathrm{~d} r_{0}^{\prime} .
\end{aligned}
$$


In Eq. (52), $M_{\psi}, M_{2}, \Omega, R$, and $\lambda$ retain their meanings as previously defined in Sects. (4.1) and (4.2), while $r_{0}$ is now the radius of the equipotential surface at the angles $\left(\theta_{0}, \phi_{0}\right)$ defined such that

$$
\begin{aligned}
& -A\left(r_{0}\right) P_{2}\left(\cos \theta_{0}\right)+B\left(r_{0}\right) P_{2}\left(\lambda_{0}\right)+C\left(r_{0}\right) P_{3}\left(\lambda_{0}\right) \\
& \quad+D\left(r_{0}\right) P_{4}\left(\lambda_{0}\right)=0,
\end{aligned}
$$

where $A\left(r_{0}\right)$ is given by Eq. (33), and $B\left(r_{0}\right), C\left(r_{0}\right)$, and $D\left(r_{0}\right)$ are defined in Eqs. (47).

Since, as previously mentioned, the distortions arising from both rotation and tidal effects are simply additive (to first- and even second-order accuracy; Kopal 1960, 1974, 1989), the external equipotential surface of the distorted star can be described by

$r\left(r_{0}, \theta, \phi\right)=r_{0}\left[1+Y_{\text {rot }}+\sum_{2}^{4} Y_{j}\right]$,

where $Y_{\text {rot }}$ is given by Eq. (28) and the $Y_{j}$ are given by Eq. (41). As in Sects. 4.1 and 4.2, the evaluation of $\eta_{j}$ can be done by numerically integrating Radau's equation (Eq. (44)). In this way, by using an adequate iterative procedure, we can obtain a value for $\eta_{2}$ that reflects the combined effects of rotation and tides, and $\eta_{3}$ and $\eta_{4}$ values that obviously relate only to tidal effects.

If we define the radial parts of the tesseral harmonics, $A\left(r_{0}\right)$, $B\left(r_{0}\right), C\left(r_{0}\right)$ and $D\left(r_{0}\right)$, according to Eqs. (33) and (47), Eq. (54) can be rewritten as

$$
\begin{aligned}
r\left(r_{0}, \theta, \phi\right)= & r_{0}\left[1-A\left(r_{0}\right) P_{2}(\cos \theta)+B\left(r_{0}\right) P_{2}(\lambda)\right. \\
& \left.+C\left(r_{0}\right) P_{3}(\lambda)+D\left(r_{0}\right) P_{4}(\lambda)\right] .
\end{aligned}
$$

By integrating Eq. (55) from $r=0$ to $r\left(r_{0}, \theta, \phi\right)$, we obtain

$$
\begin{aligned}
V_{\psi}= & \frac{4 \pi r_{0}^{3}}{3}\left[1+\frac{3 A^{2}}{5}+\frac{3 A B}{5}+\frac{3 B^{2}}{5}+\frac{3 C^{2}}{7}-\frac{2 A^{3}}{35}\right. \\
& -\frac{3 A^{2} B}{35}+\frac{9 A^{2} D}{140}+\frac{3 A B^{2}}{35}+\frac{2 A C^{2}}{35}+\frac{2 B^{3}}{35} \\
& +\frac{10 A D^{2}}{231}+\frac{6 A B D}{35}+\frac{6 B^{2} D}{35}+\frac{4 B C^{2}}{35} \\
& \left.+\frac{6 C^{2} D}{77}+\frac{20 B D^{2}}{231}+\frac{18 D^{3}}{1001}+\frac{D^{2}}{3}\right] .
\end{aligned}
$$

For simplicity, $A\left(r_{0}\right), B\left(r_{0}\right), C\left(r_{0}\right), D\left(r_{0}\right)$ appear with no arguments. From Eq. (56), $r_{0}$ and $r_{\psi}$ are related as

$$
\begin{aligned}
r_{\psi}= & r_{0}\left[1+\frac{3 A^{2}}{5}+\frac{3 A B}{5}+\frac{3 B^{2}}{5}+\frac{3 C^{2}}{7}-\frac{2 A^{3}}{35}\right. \\
& -\frac{3 A^{2} B}{35}+\frac{9 A^{2} D}{140}+\frac{3 A B^{2}}{35}+\frac{2 A C^{2}}{35}+\frac{2 B^{3}}{35} \\
& -\frac{3 A^{2} B}{35}+\frac{9 A^{2} D}{140}+\frac{3 A B^{2}}{35}+\frac{2 A C^{2}}{35}+\frac{2 B^{3}}{35} \\
& \left.+\frac{6 C^{2} D}{77}+\frac{20 B D^{2}}{231}+\frac{18 D^{3}}{1001}+\frac{D^{2}}{3}\right]^{\frac{1}{3}} .
\end{aligned}
$$

As before, $r_{0}$ can be calculated through Eq. (57) by means of an iterative procedure.

The local effective gravity is given by Eq. (51), $g$ can be numerically found by differentiation of Eq. (52). With the values of $\langle g\rangle$ and $\left\langle g^{-1}\right\rangle$ known for a set of points on an equipotential surface, $S_{\psi}\langle g\rangle$ and $S_{\psi}\left\langle g^{-1}\right\rangle$ can be found, respectively, from Eqs. (12) and (13) by numerically integrating over $\theta$ and $\phi$.

\subsection{Rotational inertia}

Rotational inertia (or moment of inertia) is an important tool for studying tidal evolution theories, since it is required to predict the circularization and synchronization time scales (Zahn 1977).

Motz (1952) computed values of rotational inertia by using the existing (and unrealistic) models at that time. Ruciński (1988) obtained values of the radii of gyration for polytropic models of low-mass stars at the ZAMS. The radius of gyration of a body is the distance between a given axis of this body ${ }^{2}$ and its center of gyration ${ }^{3}$, and is defined by

$\beta=\sqrt{\frac{I}{M R^{2}}}$

where $I$ is the rotational inertia of the star, and $M$ and $R$ are the stellar mass and radius, respectively. Claret \& Giménez (1989b) presented radii of gyration calculations for more massive stars during the hydrogen burning phases. They used standard models, in which the stars are described by spherically symmetric configurations. More recent computations of radii of gyration were provided by C04, C05, C06b, and C07.

As a consequence of the distortions introduced by rotation and tides, the rotational inertia of the star is changed from that one corresponding to spherical symmetry. Law (1980) derived the rotational inertia of a rotationally distorted mass shell, to first-order accuracy, as

$\Delta I=\frac{2}{3} \mathrm{~d} m_{\psi} r_{\psi}^{2}\left(\frac{r_{0}}{r_{\psi}}\right)^{4}\left[1+\frac{3}{20} \sum_{i=1}^{5} \alpha_{i} A^{i}\left(i \eta_{2}+5\right)\right]$,

where

$\alpha_{i}=\frac{5}{i !(5-i) !} \int_{0}^{\pi} P_{2}^{i}(\cos \theta) \sin ^{3} \theta \mathrm{d} \theta$.

In what follows, we present a new expression for the rotational inertia taking into account both rotational and tidal distortions, again considering only first-order effects. We start by considering a very thin mass shell, for which the rotational inertia is given by

$\Delta I=\int R^{2} \mathrm{~d} m$

By using spherical coordinates, so that $R=r \sin \theta$ and $\mathrm{d} m=$ $\rho r^{2} \sin \theta \mathrm{d} r \mathrm{~d} \theta \mathrm{d} \phi$, and assuming that $\rho$ is constant in such a thin mass shell (which will be later justified as we get to the limiting differential case), the previous expression becomes

$$
\begin{aligned}
\Delta I & =\rho \int_{0}^{2 \pi} \int_{0}^{\pi} \int_{r_{1}}^{r_{2}} r^{4} \sin ^{3} \theta \mathrm{d} r \mathrm{~d} \theta \mathrm{d} \phi \\
& =\frac{\rho}{5} \int_{0}^{2 \pi} \int_{0}^{\pi}\left(r_{2}^{5}-r_{1}^{5}\right) \sin ^{3} \theta \mathrm{d} \theta \mathrm{d} \phi,
\end{aligned}
$$

2 In a rotating body, the rotation axis is considered. If no axis is specified, the centroidal axis, which is the line joining the centroid of each cross section along the length of an axial member such as truss diagonal, is assumed.

3 The center of gyration of a body is defined as that point at which the whole mass might be concentrated (theoretically) without altering the body's rotational inertia. In other words, this is the center about which the body can rotate without moving linearly or vibrating. 
where $r_{1}$ and $r_{2}$ are respectively the inner and outer radius of the mass shell. For a tidally and rotationally distorted mass shell, $r_{1}$ and $r_{2}$ correspond to the radius of the equipotential surfaces of the distorted configuration as given by Eq. (55), so that

$$
\begin{aligned}
r_{i}= & r_{0 i}\left[1-A\left(r_{0 i}\right) P_{2}(\cos \theta)+B\left(r_{0 i}\right) P_{2}(\lambda)\right. \\
& \left.+C\left(r_{0 i}\right) P_{3}(\lambda)+D\left(r_{0 i}\right) P_{4}(\lambda)\right],
\end{aligned}
$$

where $i=1,2$.

Since the terms $B\left(r_{0 i}\right), C\left(r_{0 i}\right)$ and $D\left(r_{0 i}\right)$, in Eq. (63), are proportional to $\left(r_{0 i} / R\right)^{j}$ with $j=2,3,4$ respectively, and that, furthermore, they would be raised to the fifth power when substituted in Eq. (62), it follows that $B\left(r_{0 i}\right)$ would dominate over $C\left(r_{0 i}\right)$ and $D\left(r_{0 i}\right)$ in the latter equation, as $r_{0 i} / R<1$. This, in short, means that terms of order $j$ higher than 2 do not contribute significantly to the total departure from the spherical symmetry, as already noted by Claret \& Willems (2002). So, as a further approximation, we drop the terms corresponding to $j=3,4$ in Eq. (63) for the sole purpose of computing the rotational inertia of a mass shell distorted by tides and rotation. This approximation allows us to reduce from 252 to 42 the terms that result from the full development of Eq. (62) when $r_{1}$ and $r_{2}$ are substituted for those given by Eq. (63). In this way, $\Delta I$ becomes, after some algebra,

$$
\begin{aligned}
\Delta I= & \frac{\rho}{5} \int_{0}^{2 \pi} \int_{0}^{\pi}\left\{\sum _ { i = 1 } ^ { 2 } ( - 1 ) ^ { i } r _ { 0 i } ^ { 5 } \left[1-A\left(r_{0 i}\right) P_{2}(\cos \theta)\right.\right. \\
& \left.\left.+B\left(r_{0 i}\right) P_{2}(\lambda)\right]^{5}\right\} \sin ^{3} \theta \mathrm{d} \theta \mathrm{d} \phi .
\end{aligned}
$$

The fifth power term within brackets in Eq. (64) can be expanded by using the multinomial theorem

$$
\begin{aligned}
& \left(x_{1}+x_{2}+\ldots+x_{\mathrm{p}}\right)^{n}= \\
& \sum_{\substack{0 \leq a_{1}, a_{2}, \ldots, a_{\mathrm{p}} \leq n \\
a_{1}+a_{2}+\ldots+a_{\mathrm{p}}=n}}\left(\begin{array}{c}
n \\
a_{1}, a_{2}, \ldots, a_{\mathrm{p}}
\end{array}\right) x_{1}^{a_{1}} x_{2}^{a_{2}} \ldots x_{\mathrm{p}}^{a_{\mathrm{p}}},
\end{aligned}
$$

yielding

$$
\Delta I=\frac{\rho}{5} \sum_{\substack{0 \leq a_{1}, a_{2}, a_{3} \leq 5 \\ a_{1}+a_{2}+a_{3}=5}} Q_{a}\left[\sum_{i=1}^{2}(-1)^{i+1} r_{0 i}^{5} A^{a_{2}}\left(r_{0 i}\right) B^{a_{3}}\left(r_{0 i}\right)\right],
$$

where

$Q_{a}=\left(\frac{5 !}{a_{1} ! a_{2} ! a_{3} !}\right) \int_{0}^{2 \pi} \int_{0}^{\pi} P_{2}^{a_{2}}(\cos \theta) P_{2}^{a_{3}}(\lambda) \sin ^{3} \theta \mathrm{d} \theta \mathrm{d} \phi$.

For the limiting case of a thin mass shell, we have

$$
\begin{aligned}
r_{01} & =r_{0}, \\
r_{02} & =r_{01}+\mathrm{d} r_{01}=r_{0}+\mathrm{d} r_{0}, \\
A\left(r_{01}\right) & =A, \\
B\left(r_{01}\right) & =B, \\
A\left(r_{02}\right) & =A\left(r_{01}\right)+\mathrm{d} A\left(r_{01}\right)=A+\mathrm{d} A \text { and } \\
B\left(r_{02}\right) & =B\left(r_{01}\right)+\mathrm{d} B\left(r_{01}\right)=B+\mathrm{d} B,
\end{aligned}
$$

so that the terms within brackets in Eq. (66) can be rewritten as

$$
\begin{aligned}
& {\left[-r_{02}^{5} A^{a_{2}}\left(r_{02}\right) B^{a_{3}}\left(r_{02}\right)+r_{01}^{5} A^{a_{2}}\left(r_{01}\right) B^{a_{3}}\left(r_{01}\right)\right]=} \\
& {\left[-\left(r_{0}+\mathrm{d} r_{0}\right)^{5}(A+\mathrm{d} A)^{a_{2}}(B+\mathrm{d} B)^{a_{3}}+r_{0}^{5} A^{a_{2}} B^{a_{3}}\right] .}
\end{aligned}
$$

For a thin mass shell one has $r_{0} \gg \mathrm{d} r_{0}$ and consequently $A \gg \mathrm{d} A$ and $B \gg \mathrm{d} B$, so the right side of Eq. (68) can be further reduced to

$$
-r_{0}^{4} \mathrm{~d} r_{0} A^{a_{2}} B^{a_{3}}\left[a_{2} \eta_{2}^{(\mathrm{rot})}+a_{3} \eta_{2}^{(\mathrm{tid})}+5\right]
$$

where $\eta_{2}^{\text {(rot) }}$ and $\eta_{2}^{\text {(tid) }}$ stand for the $\eta_{2}$ values that would result from pure rotation or pure tidal effects, respectively.

By using this latter result and remembering that $\mathrm{d} m_{\psi}=$ $4 \pi \rho r_{\psi}^{2} \mathrm{~d} r_{\psi}$, Eq. (66) can be brought to the form

$$
\begin{aligned}
\Delta I= & -\frac{1}{20 \pi} \mathrm{d} m_{\psi} \frac{r_{0}^{4}}{r_{\psi}^{2}} \frac{\mathrm{d} r_{0}}{\mathrm{~d} r_{\psi}} \\
& \times \sum_{\substack{0 \leq a_{1}, a_{2}, a_{3} \leq 5 \\
a_{1}+a_{2}+a_{3}=5}} Q_{a} A^{a_{2}} B^{a_{3}}\left[a_{2} \eta_{2}^{(\mathrm{rot})}+a_{3} \eta_{2}^{(\mathrm{tid})}+5\right],
\end{aligned}
$$

where $Q_{a}$ is given by Eq. (67).

In the special situation in which only tidal forces are present, the rotational inertia of a given mass shell is

$\Delta I=\frac{4}{3} \mathrm{~d} m_{\psi} r_{\psi}^{2}\left(\frac{r_{0}}{r_{\psi}}\right)^{4} \frac{\mathrm{d} r_{0}}{\mathrm{~d} r_{\psi}}\left[1+\frac{3}{80 \pi} \sum_{p=1}^{5} Q_{\mathrm{p}} B^{\mathrm{p}}\left(p \eta_{2}+5\right)\right]$

where $\eta_{2}$ corresponds unambiguously to $\eta_{2}^{(\mathrm{tid})}$ and $Q_{\mathrm{p}}$ is given by

$Q_{\mathrm{p}}=\frac{5}{p !(5-p) !} \int_{0}^{2 \pi} \int_{0}^{\pi} P_{2}^{p}(\lambda) \sin ^{3} \theta \mathrm{d} \theta \mathrm{d} \phi$.

\section{Models}

Our new version of the ATON evolutionary code has not only many updated and modern features, regarding the physics of stellar interior (Ventura et al. 1998), but, also, is able to reproduce stars with spherically symmetric configurations, as well as tidally and rotationally distorted stars (see Sect.4). The grids cover a mass range from 0.09 to $3.8 M_{\odot}$ and were computed from early stages of pre-MS phase up to the main sequence. The radiative opacities are taken from Iglesias \& Rogers (1993), extended by Alexander \& Ferguson (1994) tables in the low-temperature regime. The OPAL equation of state (Rogers et al. 1996) is used in the range $3.7<\log T<8.7$, while in the low- $T$ high density regime we use the equation of state of Mihalas et al. (1988). The nuclear network includes 14 elements and 22 reactions; the relevant cross-sections are taken from Caughlan \& Fowler (1988). We adopted the solar metallicity $(Z=0.0175, Y=0.27)$. The classical Mixing Length Theory (MLT, Böhm-Vitense 1958) was used to treat the convective transport of energy, though the ATON code can also use the FST (Full Spectrum of Turbulence) treatment (Canuto et al. 1996). The mixing length parameter has been set to $\alpha=\Lambda / H_{\mathrm{p}}=1.5$, value that, according to our calibration, best reproduces the solar radius at the solar age with boundary conditions obtained from gray atmosphere models (Landin et al. 2006).

We present four sets of evolutionary models, namely: (i) standard, spherical models (with no distorting effect) corresponding to single non-rotating stars; (ii) binary models (distorted only by tidal forces) in which we treat non-rotating stars in binary systems; (iii) rotating models (distorted only by rotation) for representing single rotating stars and finally; (iv) rotating binary models (distorted simultaneously by rotation and tidal forces), useful to study rotating stars in binary systems. In cases 
Table 1. Pre-MS evolutionary tracks (including $\log k_{j}$ and $\beta$ ) for $1 M_{\odot}$ star generated with our standard models ${ }^{a}$.

\begin{tabular}{rrrrrrrc}
\hline \hline $\log$ Age $(\mathrm{yr})$ & $\log \frac{L}{L_{\odot}}$ & $\log T_{\text {eff }}(\mathrm{K})$ & $\log g(\mathrm{cgs})$ & $\log k_{2}$ & $\log k_{3}$ & $\log k_{4}$ & $\beta$ \\
\hline 2.2954 & 1.8909 & 3.5902 & 1.8604 & -0.9075 & -1.3965 & -1.7387 & 0.4177 \\
3.6550 & 1.6727 & 3.6057 & 2.1405 & -0.8654 & -1.3526 & -1.6927 & 0.4253 \\
4.1464 & 1.4526 & 3.6185 & 2.4119 & -0.8325 & -1.3076 & -1.6379 & 0.4319 \\
4.5435 & 1.2322 & 3.6293 & 2.6755 & -0.8060 & -1.2707 & -1.5920 & 0.4374 \\
5.1029 & 1.0251 & 3.6376 & 2.9159 & -0.7868 & -1.2434 & -1.5574 & 0.4415 \\
5.3018 & 0.8982 & 3.6418 & 3.0597 & -0.7776 & -1.2301 & -1.5405 & 0.4435 \\
5.5216 & 0.6905 & 3.6472 & 3.2889 & -0.7664 & -1.2139 & -1.5197 & 0.4459 \\
5.7953 & 0.4699 & 3.6510 & 3.5245 & -0.7587 & -1.2028 & -1.5053 & 0.4476 \\
6.0918 & 0.2493 & 3.6526 & 3.7517 & -0.7535 & -1.1954 & -1.4958 & 0.4488 \\
6.3961 & 0.0286 & 3.6516 & 3.9682 & -0.7769 & -1.2180 & -1.5174 & 0.4495 \\
6.7156 & -0.1866 & 3.6497 & 4.1757 & -0.7904 & -1.2420 & -1.5475 & 0.4458 \\
7.0039 & -0.3157 & 3.6560 & 4.3301 & -0.8492 & -1.3026 & -1.6126 & 0.4260 \\
7.2246 & -0.2773 & 3.6840 & 4.4038 & -1.0944 & -1.5458 & -1.8532 & 0.3811 \\
7.3696 & -0.1155 & 3.7255 & 4.4078 & -1.5590 & -2.0471 & -2.3655 & 0.3202 \\
7.4906 & -0.0389 & 3.7573 & 4.4585 & -1.8961 & -2.4599 & -2.8086 & 0.2812 \\
8.7585 & -0.1301 & 3.7516 & 4.5270 & -1.7105 & -2.2631 & -2.6077 & 0.2974 \\
9.3538 & -0.0815 & 3.7553 & 4.4933 & -1.8011 & -2.3570 & -2.7027 & 0.2872 \\
9.5876 & -0.0307 & 3.7587 & 4.4558 & -1.9017 & -2.4620 & -2.8096 & 0.2767 \\
9.6979 & 0.0084 & 3.7608 & 4.4252 & -1.9810 & -2.5432 & -2.8916 & 0.2689 \\
9.8058 & 0.0619 & 3.7627 & 4.3793 & -2.0934 & -2.6579 & -3.0078 & 0.2588 \\
\hline
\end{tabular}

${ }^{a}$ The complete version of the table, including 88 tracks for the four sets of models and all the masses of Table 2, is available only in electronic form at the CDS. The tracks corresponding to rotating models and rotating binary models will contain an additional column regarding the rotational period.

in which rotation is present, we assume rigid body rotation. The relation between initial angular momentum $\left(J_{\text {in }}\right)$ and stellar mass that we used was obtained from the respective mass-radius and mass-moment of inertia relations from Kawaler (1987):

$J_{\text {kaw }}=1.566 \times 10^{50}\left(\frac{M}{M_{\odot}}\right)^{0.985} \mathrm{cgs}$.

For computing the binary models (both rotating and nonrotating) we assumed a separation of 7 times the radius of the star whose evolution is followed (from this point on referred to as the primary), which is in the range typical for close binary systems (Hilditch 2001); the disturbing star is considered to be a point mass of the same mass as its primary. As previously stated, the pseudo-potential centrifugal terms arising from the orbital motion around the system's center of mass are not included in the present approximation for the tidal effects due to a companion. We followed the evolution of internal structure constants and moment of inertia during the pre-MS phase and tabulated them together with the corresponding evolutionary tracks. Table 1 presents the $1 M_{\odot}$ standard model as an example of such tables. Column 1 gives the logarithm of stellar age (in years); Col. 2, the logarithm of stellar luminosity (in solar units); Col. 3, the logarithm of effective temperature (in K); and Col. 4, the logarithm of effective gravity (in cgs). Columns 5-7 give the logarithm of internal structure constants, and Col. 8 gives the gyration radius.

Table 2 presents the internal structure constants and the gyration radius at the ZAMS, for both non-rotating and rotating models. Its successive columns give the stellar mass (in $M_{\odot}$ ); the logarithm of the stellar luminosity (in solar units); the logarithm of the effective temperature (in $\mathrm{K}$ ); the logarithm of the surface gravity (in cgs units); the logarithm of the internal structure constants $k_{2}, k_{3}$ and $k_{4}$; the radius of gyration $\beta$ (cf. Eq. (58)); and, finally, the rotational periods (in days), when rotation is present.

\section{Results and discussion}

In this section, we discuss the results yielded by the models presented in Sect. 5 and the differences obtained with each set of models. Then we proceed with a comparison with other works in the literature.

\subsection{Effects on the internal structure constants}

As previously mentioned, Mohan et al. (1990) found that the effects of rotational distortions on main-sequence stellar models are greater than those of tidal distortions, though their results were obtained only for binary models with a mass ratio of 0.1 . We investigated the ZAMS models in order to verify such differences, by calculating how different the non-standard values of the second-order internal structure constants are as compared with the standard ones for each stellar mass at the ZAMS. The binary models produced values of $\log k_{2}$ lower than the standard models, on average, by a difference of about 0.0170 , with a maximum difference of 0.1330 occurring for the $0.9 M_{\odot}$ model. For the rotating and the rotating binary models this average difference is 0.0570 and 0.0594 , respectively; the maximum difference happens at the mass of $1.6 M_{\odot}$ for both sets of models, being 0.1603 for rotating models and 0.1654 for rotating binary ones.

To better assess the relative importance of the rotational and tidal effects, we also ran rotating binary models of 0.3 and 1.0 solar masses with (a) separations of 3, 14, 35 and 70 times the radius of the primary, (b) masses of the disturbing star corresponding to the double and half values of the primary mass, and (c) initial angular momentum equal to 0,1 and $3 J_{\text {kaw }}$, where $J_{\text {kaw }}$ is given in Eq. (72). Table 3 presents, from left to right, the primary mass, the initial angular momentum, the secondary mass, the separation between the components and the logarithm of $k_{2}$ at the ZAMS for these additional models. From that table we can see that, with the only exception of the case of $1 M_{\odot}$ with orbital separation of 3 times the primary's radius and $J_{\text {in }}$ equal to $J_{\text {kaw }}, \log k_{2}$ is mainly affected by the inclusion of 
Table 2. Internal structure constants and gyration radii for ZAMS models. See the text for details.

\begin{tabular}{|c|c|c|c|c|c|c|c|c|c|c|c|c|c|c|c|c|c|}
\hline $\begin{array}{c}M \\
\left(M_{\odot}\right) \\
\end{array}$ & $\log \frac{L}{L_{\odot}}$ & $\begin{array}{l}\log T_{\text {eff }} \\
\text { (K) }\end{array}$ & $\begin{array}{l}\log g \\
(\mathrm{cgs})\end{array}$ & $\log k_{2}$ & $\log k_{3}$ & $\log k_{4}$ & $\beta$ & $\begin{array}{l}P \\
\text { (d) }\end{array}$ & $\begin{array}{c}M \\
\left(M_{\odot}\right)\end{array}$ & $\log \frac{L}{L_{\odot}}$ & $\log T_{\text {eff }}$ & $\log g$ & $\log k_{2}$ & $\log k_{3}$ & $\log k_{4}$ & $\beta$ & $\begin{array}{l}P \\
\text { (d) }\end{array}$ \\
\hline \multicolumn{9}{|c|}{ Standard (spherically symmetric) models } & \multicolumn{9}{|c|}{ Tidally (non-rotating) distorted models } \\
\hline 0.09 & -3.3197 & 3.4367 & 5.4113 & -0.8134 & -1.2455 & -1.5272 & 0.4600 & - & 0.09 & -3.3260 & 3.4355 & 5.4146 & -0.8173 & -1.2500 & -1.5318 & 0.4601 & - \\
\hline 0.10 & 3.0098 & & 3360 & -0.8359 & -1.2743 & -1.5613 & 0.4554 & - & 0.10 & -3.0131 & 3.4836 & 5.3398 & -0.8391 & -1.2779 & -1.5651 & 0.4556 & - \\
\hline 0.20 & -2.2529 & 3.5255 & 5.0462 & -0.8943 & -1.3520 & -1.6567 & 0.4453 & - & 0.20 & -2.2540 & 3.5255 & 5.0490 & -0.9000 & -1.3592 & -1.6651 & 0.4454 & - \\
\hline 0.30 & -1.9334 & 3.5444 & 4.9788 & -0.8720 & -1.3253 & -1.6269 & 0.4489 & - & 0.30 & -1.9344 & 3.5445 & 4.9815 & -0.8770 & -1.3317 & -1.6345 & 0.4490 & - \\
\hline 0.40 & -1.6724 & 3.5626 & 4.9153 & -0.9176 & -1.3812 & -1.6922 & 0.4402 & - & 0.40 & -1.6716 & 3.5628 & 4.9168 & -0.9213 & -1.3870 & -1.6995 & 0.4397 & - \\
\hline 0.50 & -1.3820 & 3.5885 & 4.8254 & -1.0460 & -1.5023 & -1.8099 & 0.4119 & - & 0.50 & -1.3815 & 3.5888 & 4.8275 & -1.0501 & -1.5082 & -1.8173 & 0.4114 & - \\
\hline 0.60 & -1.0632 & 3.6252 & 4.7325 & -1.2494 & -1.7065 & -2.0127 & 0.3808 & - & 0.60 & -1.0598 & 3.6240 & 4.7260 & -1.2309 & -1.6897 & -1.9975 & 0.3821 & - \\
\hline 0.70 & -0.7584 & 3.6639 & 4.6496 & -1.4603 & -1.9300 & -2.2404 & 0.3534 & - & 0.70 & -0.7558 & 3.6640 & 4.6487 & -1.4621 & -1.9334 & -2.2449 & 0.3532 & - \\
\hline 0.80 & 1808 & 3.7026 & 4.5849 & -1.6441 & -2.1288 & -2.4442 & 0.3314 & - & 0.80 & -0.4778 & 3.7024 & 4.5825 & -1.6417 & -2.1282 & -2.4453 & 0.3312 & - \\
\hline 0.90 & -0.3454 & 3.7280 & 4.6022 & -1.7067 & -2.2259 & -2.5554 & 0.3216 & - & 0.90 & -0.2348 & 3.7312 & 4.5059 & -1.8397 & -2.3484 & -2.6735 & 0.3071 & - \\
\hline 1.00 & -0.1253 & 3.7540 & 4.5319 & -1.9037 & -2.4571 & -2.8011 & 0.2957 & - & 1.00 & -0.1228 & 3.7546 & 4.5331 & -1.9185 & -2.4769 & -2.8234 & 0.2950 & - \\
\hline 1.20 & 0.3184 & 3.7935 & 4.3251 & -2.4573 & -3.1435 & -3.5662 & 0.2361 & - & 1.20 & 0.3319 & 3.7941 & 4.3156 & -2.4968 & -3.1894 & -3.6146 & 0.2334 & - \\
\hline 1.40 & 0.6830 & 3.8214 & 4.1394 & -2.8859 & -3.7014 & -4.2406 & 0.1962 & - & 1.40 & 0.6901 & 3.8219 & 4.1355 & -2.9077 & -3.7278 & -4.2709 & 0.1950 & - \\
\hline 1.60 & & 3.8590 & 4.0749 & -2.9992 & & -4.4404 & 0.1861 & - & 1.60 & 0.9630 & 595 & 4.0709 & -3.0239 & -3.8827 & -4.4670 & 0.1853 & - \\
\hline 1.80 & & 3.8998 & 4.0611 & -2.9 & -3 & -4 & 0. & - & 100 & 1.1883 & 011 & 4.0629 & -3.0035 & -3 & -4 & 0.1866 & \\
\hline 2.00 & 97 & 3.9357 & & -2.9667 & 03 & -4.4044 & & - & 2.0 & & & 4.0541 & -2.9 & -3 & -4.4182 & & - \\
\hline 2.30 & 1.6345 & 3.9811 & 4.0419 & -2.9337 & -3.7823 & -4.3644 & 0.1 & - & 2.30 & 1.6376 & 826 & 4.0464 & -2.9370 & -3.7882 & -4.3727 & 0.1912 & - \\
\hline 2.50 & & 4.0073 & & -2.9098 & -3 & -4 & 930 & - & 2.50 & 1.7867 & 089 & & -2.9233 & -3 & -4.3542 & 0.1930 & - \\
\hline 2.80 & 82 & & & -2.8 & & & 967 & - & 2.80 & 1.9834 & 147 & & -2 & -3. & -4 & 0.1962 & - \\
\hline 2.00 & 2 & 4. & 96 & -2.8 & -3 & -4 & 0 & - & 3.0 & 2.1002 & 64 & 14 & -2 & -3 & -4 . & & - \\
\hline 3 . & 587 & 4.0938 & 4.0257 & -2.8049 & -3 & 186 & 0.2014 & - & 3.3 & 2.2589 & 9959 & 4.0350 & 987 & -3.6360 & -4.2145 & 0.2018 & - \\
\hline 3. & 2.3542 & 4.1121 & 4.0287 & -2 & -3 & -4 & 0. & - & 3.50 & 2.3593 & 28 & 75 & -2 & -3 & -4 & 32 & \\
\hline & 4869 & 4.1368 & 4.0307 & -2.7394 & -3.5731 & -4 & 0.2069 & - & 3.80 & 15 & 4.1378 & 4.0313 & -2.7537 & -3.5871 & -4.1636 & 0.2065 & - \\
\hline \multicolumn{9}{|c|}{ Rotationally distorted (single star) models } & \multicolumn{9}{|c|}{ Rotationally and tidally distorted models } \\
\hline 0.09 & 403 & 3.3830 & 5.4499 & -0.8321 & -1.2660 & -1.5486 & 465 & $\overline{03}$ & 0.09 & 3.5459 & 3.3818 & 5.4520 & -0.8317 & -1.2657 & -1.5481 & 0.4655 & $\overline{322}$ \\
\hline 0.10 & -3.2999 & 3.4264 & 5.4644 & -0.8 & -1.3053 & -1.5923 & 0.4666 & 0237 & 0.10 & -3.3060 & 3.4251 & 5.4666 & 649 & -1.3047 & -1.5916 & 0.4664 & .0236 \\
\hline 0.20 & -2.3178 & & & & -1. & -1 . & 50 & 0430 & 0.2 & 70 & & & & -1 & & 49 & 0.0429 \\
\hline 0.30 & 9701 & 3.5350 & 52 & -0. & -1 & -1 & 520 & 7 & 0.30 & 707 & & & & -1 & & 0.4518 & 0.0775 \\
\hline 0.40 & -1.7068 & 3.5551 & 4.9499 & -0.9340 & -1.4047 & -1.7203 & 0.4465 & 0.1159 & 0.40 & -1.7041 & 3.5553 & 4.9491 & -0.9358 & -1.4072 & -1.7232 & 0.4457 & 0.1160 \\
\hline & 4194 & & & -1 & -1 & -1 & 182 & 1561 & 0.50 & -1.4171 & & & & & -1 & 0.4176 & 0.1562 \\
\hline & 005 & 3.6164 & 90 & -1.2 & -1.7069 & -2. & 52 & 995 & 0.60 & & 65 & 4.7583 & -1 & -1 & -2 & 0.3849 & 0.2001 \\
\hline & & 3.6551 & & & & & 0.3566 & 0.2445 & 0.70 & & & & -1.4540 & & & & 0.2454 \\
\hline 0.80 & 102 & & 54 & -1 & 05 & -2 & 0.3334 & 0.2845 & 0.8 & 75 & & 0 & -1 & -2 & -2 & 39 & 0.2897 \\
\hline 0.90 & 657 & 3.7 & 4.2 & -1 & 63 & -2 & 0. & 3 & 0.9 & & & 2 & -1 & -2 & -2 & 80 & 0.3247 \\
\hline & -0.1458 & 3.7465 & 4.5486 & -1.9242 & -2.4777 & -2.8225 & 0.2963 & 0.3233 & 1.00 & -0.1444 & & 95 & -1.9231 & -2 & -2 . & 0.2959 & 0.3228 \\
\hline 1.20 & & & & -2.4765 & -3.1529 & -3.5633 & 0.2362 & 0.3968 & 1.20 & 0.3355 & 3.7672 & & -2.4134 & -3.0060 & -3.3663 & 0.2432 & 0.5464 \\
\hline 1. & 679 & 3.8 & & -2 . & 73 & & 0. & 1 & 1.4 & & & 4. & -3 & & & 78 & 0.4974 \\
\hline & 0.9437 & 3.8406 & 4.0753 & -3.1595 & 570 & -4.6681 & 0.1749 & & 1.60 & 0.9492 & 3.8400 & 4.0696 & -3.1646 & -4.0 & -4 & 0.1740 & 0.5902 \\
\hline & & 3.8852 & & & 057 & -4.6137 & 0.1778 & 0.6840 & 1.80 & & & & & -4. & -4 & 0.1774 & 0.6854 \\
\hline 2. & & 3.9229 & & & & & & 0.7948 & 2.0 & & 25 & & & & & & 0.7992 \\
\hline 2.30 & 1.6297 & 3.9723 & 4.0388 & -3 & 55 & -4.4723 & 0.1858 & 0.9586 & 2.3 & 1.6333 & 3.9719 & 4.0353 & -3 & -3 & -4 & 0.1852 & 0.9638 \\
\hline & 1.7790 & 4.0003 & & & & & 0.1886 & & 2.50 & & & & & & & & 1.0762 \\
\hline & 1.9768 & & & -2.9217 & -3 . & -4.3 & 0.1927 & 1.2590 & 2.8 & 1.9787 & & & -2.9249 & & -4 . & 0.1925 & 1.2588 \\
\hline & & 4.0602 & 4.0286 & -2.8 & & -4.3279 & 0.1954 & 1.3821 & 3.0 & 2.0957 & 4.0607 & & -2.8819 & -3 & -4.3199 & 0.1954 & 1.3785 \\
\hline & & 4.0906 & & & & & 0.1993 & 1.5722 & 3.3 & & 4.0904 & & -2.8 & & 723 & 0.1988 & 1.5784 \\
\hline 3 & 2. & 4.1085 & 4.0259 & -2.8 & -3. & -4.2405 & 0.2014 & 1.7091 & 3.5 & 2.3527 & 4.1094 & 4.0311 & -2.8084 & -3. & -4.2333 & & 1.6983 \\
\hline 3.80 & 2.4866 & 4.1338 & 4.0271 & -2.7748 & 134 & -4.1936 & 0.2048 & 1.9082 & 3.80 & 2.4880 & 4.1343 & 4.0291 & -2.7711 & -3.6083 & -4.1878 & 0.2047 & 1.9038 \\
\hline
\end{tabular}

Table 3. Values of $\log k_{2}$ obtained for additional models ${ }^{b}$.

\begin{tabular}{ccccc}
\hline \hline$M_{\text {prim }}\left(M_{\odot}\right)$ & $J_{\text {in }}\left(J_{\text {kaw }}\right)$ & $M_{\text {sec }}\left(M_{\odot}\right)$ & $R\left(r_{\star}\right)$ & $\log \left(k_{2}\right)$ \\
\hline 0.30 & $\ldots$ & $\ldots .30$ & 7 & -0.872 \\
0.30 & $\ldots$ & 0.377 \\
0.30 & 1 & $\ldots .30$ & $\ldots$ & -0.921 \\
0.30 & 1 & 0.30 & 14 & -0.919 \\
0.30 & 1 & 0.918 & 35 & -0.919 \\
0.30 & 1 & 0.30 & 70 & -0.920 \\
0.30 & 1 & 0.15 & 7 & -0.918 \\
0.30 & 1 & 0.60 & 7 & -0.919 \\
0.30 & 1 & 0.30 & 3 & -0.868 \\
0.30 & $\ldots$ & 0.30 & 3 & -0.916 \\
0.30 & 1 & $\ldots$ & $\ldots$ & -1.904 \\
1.00 & $\ldots$ & 1.00 & 7 & -1.918 \\
1.00 & $\ldots$ & $\ldots$ & $\ldots$ & -1.924 \\
1.00 & 1 & 1.00 & 7 & -1.923 \\
1.00 & 1 & 1.00 & 14 & -1.926 \\
1.00 & 1 & 1.00 & 35 & -1.935 \\
1.00 & 1 & 1.00 & 70 & -1.930 \\
1.00 & 1 & 0.50 & 7 & -1.932 \\
1.00 & 1 & 2.00 & 7 & -1.939 \\
1.00 & 1 & 1.00 & 3 & -2.094 \\
1.00 & $\ldots$ & 1.00 & 3 & -2.027 \\
1.00 & 1 & 1.00 & 3 & -2.277 \\
1.00 & 3 &
\end{tabular}

${ }^{b}$ Ellipsis dots (...) indicate missing values, because the model is either non-rotating or not binary. Rows with all numbers correspond to rotating binary models.

rotational effects. For $0.3 M_{\odot}$, considering wider orbits and assuming different mass binary components do not alter significantly the $\log k_{2}$ values. For the $1 M_{\odot}$ model, $\log k_{2}$ decreases slightly when the distance between the stars increases, except for separations of 3 and 70 stellar radius, and when we vary the mass of the companion stars. The maximum difference occurs for $1 M_{\odot}$ models, when we consider a companion star of $2 M_{\odot}$.

The particular cases of $1 M_{\odot}$ with orbital separation of 3 times the primary's radius and $J_{\text {in }}=J_{\text {kaw }}$ or $J_{\text {in }}=3 J_{\text {kaw }}$ (where $J_{\text {kaw }}$ denotes the initial angular momentum given by Eq. (72)) demonstrate however that, depending on the star's rotation rate, a shorter orbital separation can make the tidal effects to overcome the rotational ones regarding the internal structure constants. This can be seen in Fig. 1, which also shows that the differences become more apparent for ages starting at the ZAMS.

For the gyration radii, we found, for a given mass at the ZAMS, values slightly higher for the rotating binary than for those calculated with the standard models. This is due to the combined differences between the radius and the rotational inertia of each model.

Concerning the effects on the internal structure of the stars, the models distorted only by tidal forces are in general similar to the standard ones and the models distorted only by rotation are similar to the rotating binary models. This is due to the fact that, for the adopted orbital separation, the rotation effects are, on average, more important than the tidal ones. Here, we concentrate in the differences between the standard models and the rotating binary models, since this is the case of scientific interest for apsidal motion studies.

In Fig. 2 we show the path followed by our standard models (solid lines) and our rotationally and tidally distorted models (dashed lines) for the masses of $0.09,0.2,0.3,0.4,0.6,0.8,1.0$, $1.2,1.8,2.0$, and $3.8 M_{\odot}$. We can see that the distorted pre-MS evolutionary tracks have lower $T_{\text {eff }}$ than their standard counterparts, as it is already known for stars distorted only by rotation. 


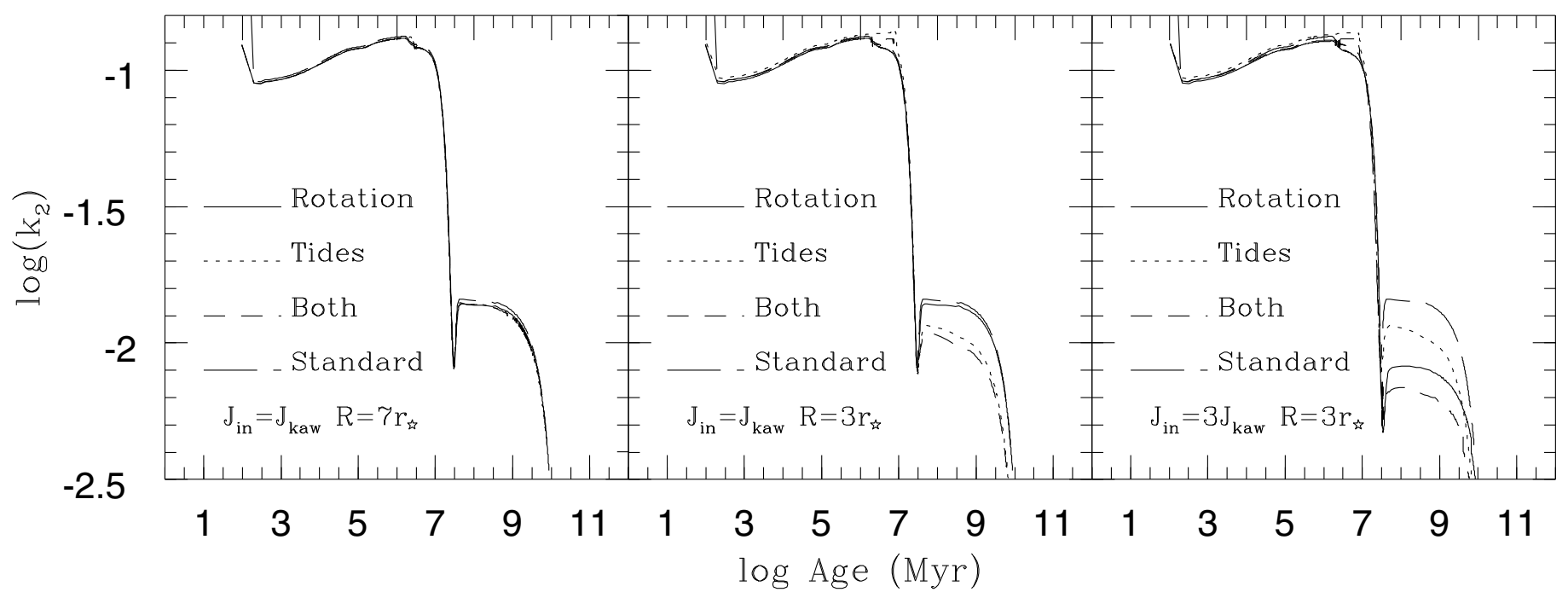

Fig. 1. $\log k_{2}$ values against age for an $1 M_{\odot}$ models with orbital separation $R$ and initial angular momentum $J_{\text {in }}$ as indicated, where $r_{\star}$ denotes the primary's radius and $J_{\text {kaw }}$ the initial angular momentum given by Kawaler's (1987) relations. Long-dashed lines represent values for the standard (non-rotating, single star) model; solid lines, the rotating-only model; dotted lines, the binary model without rotation; and the short-dashed lines, the rotating binary model.

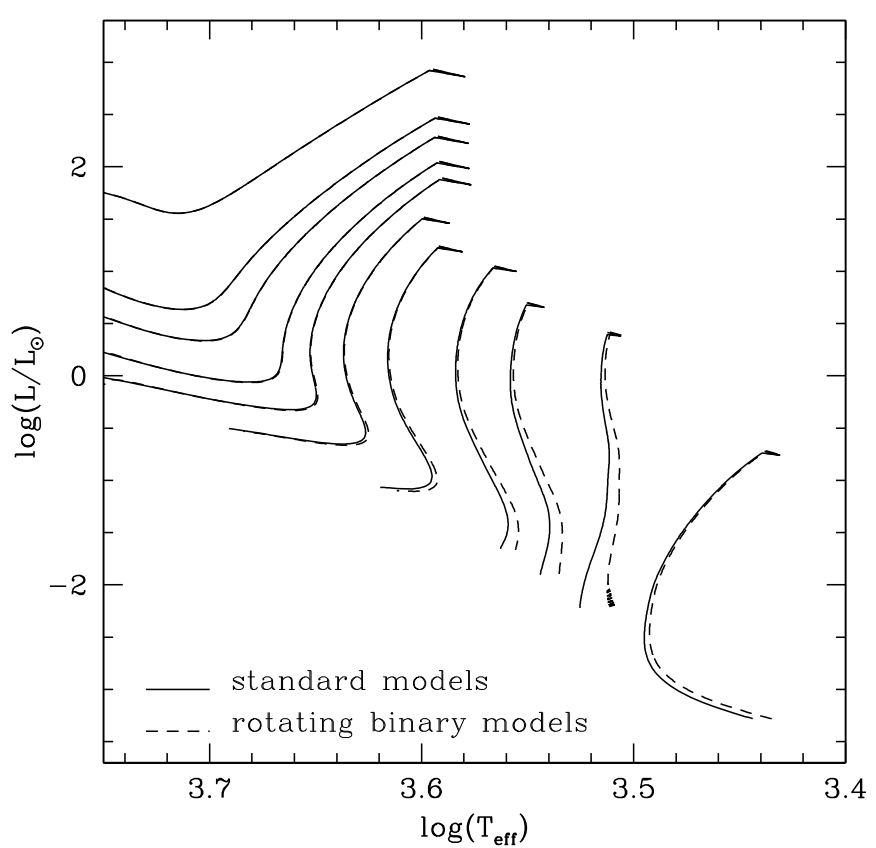

Fig. 2. Evolutionary tracks for standard and for rotating binary models. The model masses correspond to $0.09,0.20 .3,0.4,0.6,0.8,1.0,1.2$, $1.8,2.0$, and $3.8 M_{\odot}$ from bottom to top.

Sackmann (1970) reported that the distorting effects of rotation affects late-type, main-sequence stars much more seriously than the early-type ones, and that this behavior could be approximately understood by looking at the variation of the power law expressions of the nuclear-energy-generation rates along the main sequence. This trend is also observed when tidal and rotational distortions act together during the pre-MS.

Figure 3 , in which we plot $\log k_{2}$ against the stellar age for selected masses of our standard models and rotating binary models, shows the significant change in mass concentration during the pre-MS evolution, especially for higher masses. For ages less than $1 \mathrm{Myr}$ the $\log k_{2}$ do not vary significantly, neither with time nor with mass (see Fig. 3). For models with masses lower than $0.3 M_{\odot}$, that correspond to the mass range where stars are almost

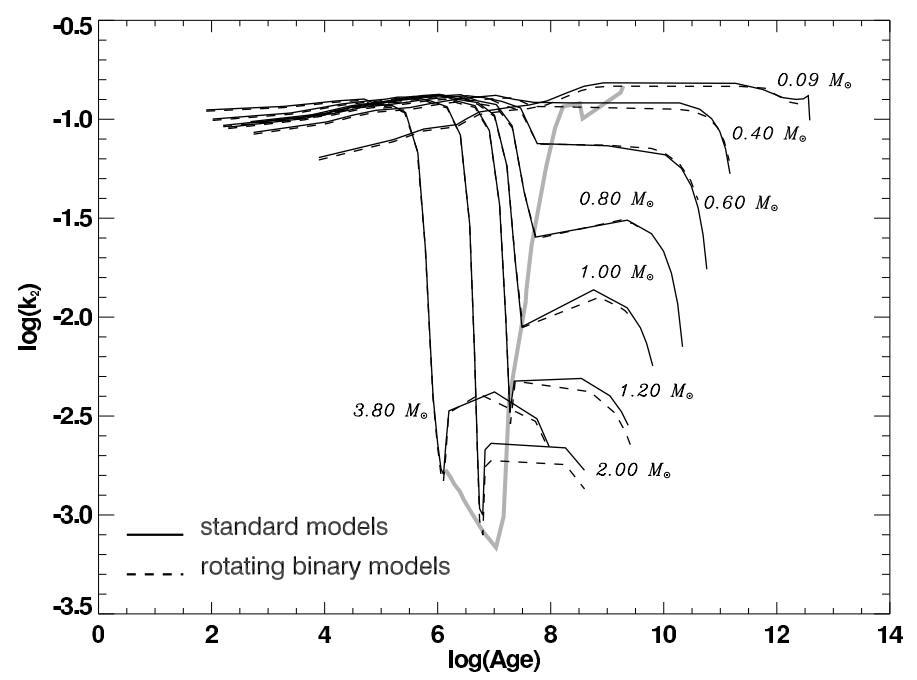

Fig. 3. The time evolution of $\log k_{2}$ for selected masses of the standard and the rotating binary models. The gray line corresponds to the ZAMS.

completely convective, $\log k_{2}$ remains roughly constant during the evolution. For masses greater than $0.3 M_{\odot}$ the values of $\log k_{2}$ are constant until a given age, after which they start to drop; the decrease of $\log k_{2}$ starts earlier as the mass increases. It seems that $\log k_{2}$ remains constant until the star develops a radiative core.

The values of $\log k_{j}$ versus stellar mass for standard models and rotating binary models at the ZAMS are shown in Fig. 4, in which we see that the distorted models corresponding curves remain below those corresponding to the standard models. The values of $\log k_{j}$ are more sensitive to the distorting effects for $M<$ $0.5 M_{\odot}$ and for $M>1.4 M_{\odot}$. The values of $\log k_{2}, \log k_{3}$, and $\log k_{4}$ remain roughly constant in the mass range $0.09-0.4 M_{\odot}$. As the mass increases from $0.4 M_{\odot}$ to $1.5 M_{\odot}, k_{j}$ drop significantly (2-3 orders of magnitude) and reach their minimum value at $1.5 M_{\odot}$. For masses greater than $1.5 M_{\odot}$ we note a parallel behavior of the $k_{j}$. H87 pointed out that, for a given mass, there is a significant decrease in $k_{j}$ for increasing $j$, and that the relevance of including the higher order terms, when comparing with observations, can be judged from Eq. (2). In our analysis $\log k_{3}$ 


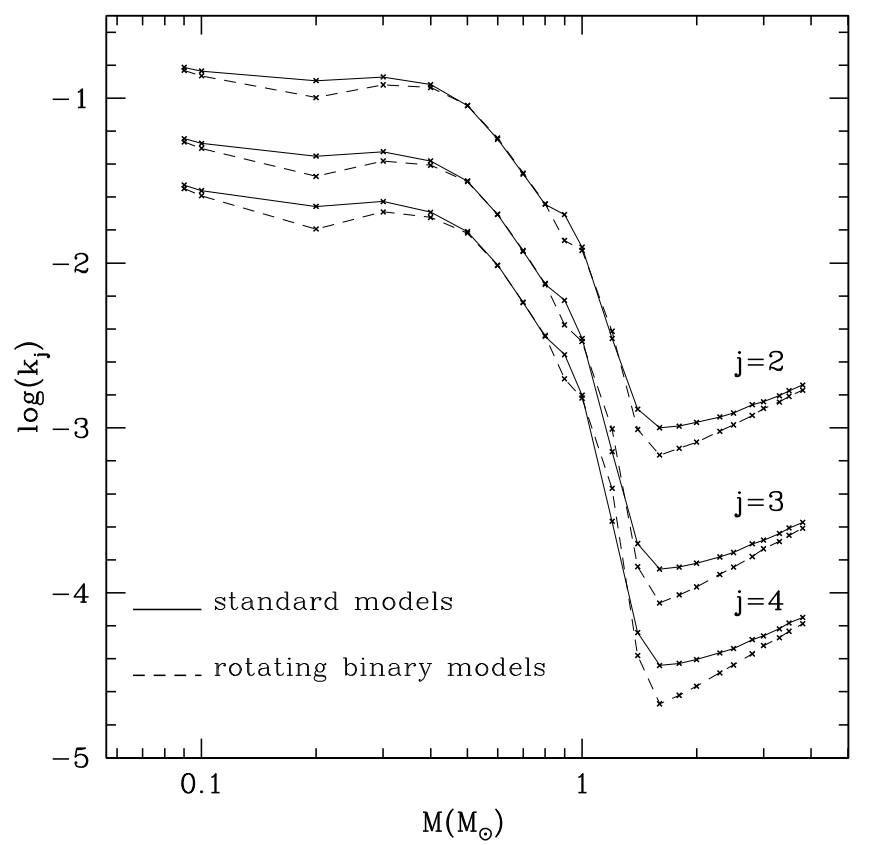

Fig. 4. $\log k_{j}$ as a function of $\log M$ for ZAMS models, for the the same set of masses as in Fig. 2.

and $\log k_{4}$ are really less important than $\log k_{2}$ in the mass range that he analyzed $\left(0.5-32 M_{\odot}\right)$, but the same statement cannot be extended to less massive stars $\left(M \leq 0.5 M_{\odot}\right)$ : as can be seen from Table 2 and also from Fig. $4, k_{4}$ is lower than $k_{2}$ by more than one order of magnitude in the mass range 1.5-3.8 $M_{\odot}$, but this difference decreases to less than one order of magnitude for stars less massive than $0.5 M_{\odot}$. So, in the low-mass range, the assumption that the harmonics of order greater than $j=2$ can be neglected, widely used when analyzing the apsidal motion of binary systems, seems not to be entirely justified, except maybe when computing the apsidal motion rate $\dot{\omega}$ in which, besides a dependence on $\log k_{j}$, there is also a dependence on $\left(r_{0} / R\right)^{j+3}$ that will make the $j=2$ term always dominate unless $r_{0}$ is very close to $R$.

In Fig. 5 we show $\log \beta$ as a function of $\log M$, at the ZAMS, for our standard and non-standard models and also for the nonrotating models by Claret \& Giménez (1989b). For masses above $1.4 M_{\odot}$ our models predict a lower value of $\log \beta$ than the Claret \& Giménez (1989b) ones; the values predicted by our rotating binary models are even lower. Although these models have slightly different chemical compositions, the comparison is still valid. A control made with a one solar mass model with the same initial chemical composition as those by Claret \& Giménez (1989b) shows that the gyration radius at the ZAMS did not vary significantly.

The time evolution of $\log \beta$ has the same behavior as the time evolution of $\log k_{j}$ (Fig. 3). Also, $\log \beta$ behaves roughly in the same way as $\log k_{j}$ as a function of $\log M$ (Fig. 4), with a minimum at $1.5 M_{\odot}$, corresponding to the change in the dominant energy source from the p-p chain to the CNO cycle (Claret \& Giménez 1989b). This similarity is due to the known linear relationship between $\log k_{j}$ and $\log \beta$, also seen in Fig. 1 by Motz (1952).

The differences of the stellar radii as a function of stellar age and mass for the standard and distorted models are shown in Fig. 6. For a $0.5 M_{\odot}$ star, it can be seen that the stellar radii at

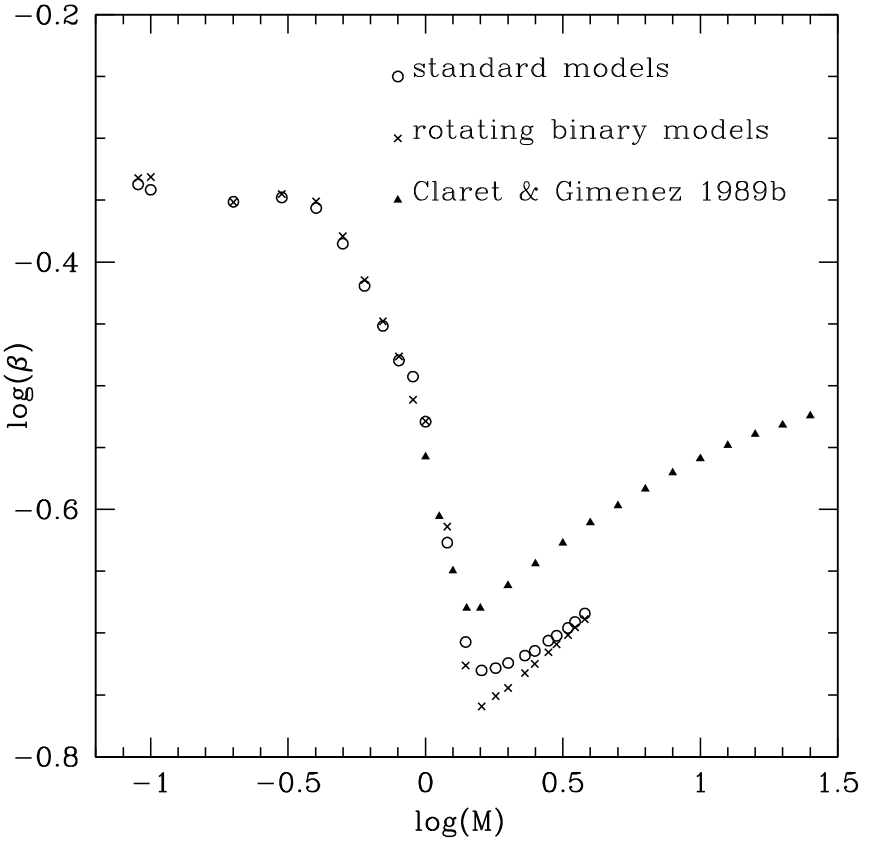

Fig. 5. Standard models (open circles) and rotating binary models (crosses) in the $\log \beta-\log M$ plane. The full triangles correspond to the results by Claret \& Giménez (1989b).

the ZAMS of the rotating binary models are slightly smaller than those produced by the standard models. For the $1 M_{\odot}$ model, the situation is the opposite: the distorted models predict a slightly larger stellar radius at the ZAMS than the standard models; this difference becomes still larger for the $2 M_{\odot}$ model. The threshold mass for this transition is at $\sim 0.7 M_{\odot}$. Sackmann (1970) had already noted this behavior, finding a threshold mass about 1.3-1.5 $M_{\odot}$ and suggested that it could be related to the crossover from the proton-proton chain to the $\mathrm{CNO}$ cycle that occurs around $1.5 M_{\odot}$, depending on the initial chemical composition. According to him, this behavior of the effects of rotation shows up in all physical quantities of a star. Figure 6 also shows the stellar radius along the mean system axis, defined as the axis joining the two stars. For all models computed the mean axis is greater than the mean stellar radius during the whole evolution, the difference being more evident for ages higher than $\sim 100 \mathrm{Myr}$ $\left(0.5 M_{\odot}\right), \sim 20 \operatorname{Myr}\left(1.0 M_{\odot}\right)$ and $\sim 10 \operatorname{Myr}\left(2.0 M_{\odot}\right)$.

As a final remark, we verified that tidal effects act in the same way as rotational ones but on a smaller scale.

\subsection{Comparison with other works}

Computed values of internal structure constants for mainsequence stars are available in the literature from other authors. We checked the validity of our calculations by comparing our results of $\log k_{2}$ at the ZAMS with those previously published ones. Our standard values of internal structure constants are in qualitative agreement with those by H87, CG89a and CG92, as well as our distorted $\log k_{2}$ relative to those given by $\mathrm{C} 04$, C05, C06 and C07. To better compare our standard results with the previous ones, we computed additional grids of $1 M_{\odot}$ standard models, with different values of the mixing length parameter ( $\alpha=2.0$ and $\alpha=1.5)$ and four different initial chemical compositions, $(X, Z)=(0.7,0.02),(0.7,0.04),(0.7,0.004)$, and $(0.8,0.02)$. From Fig. 7 , where the time-dependence of $\log k_{2}$ 

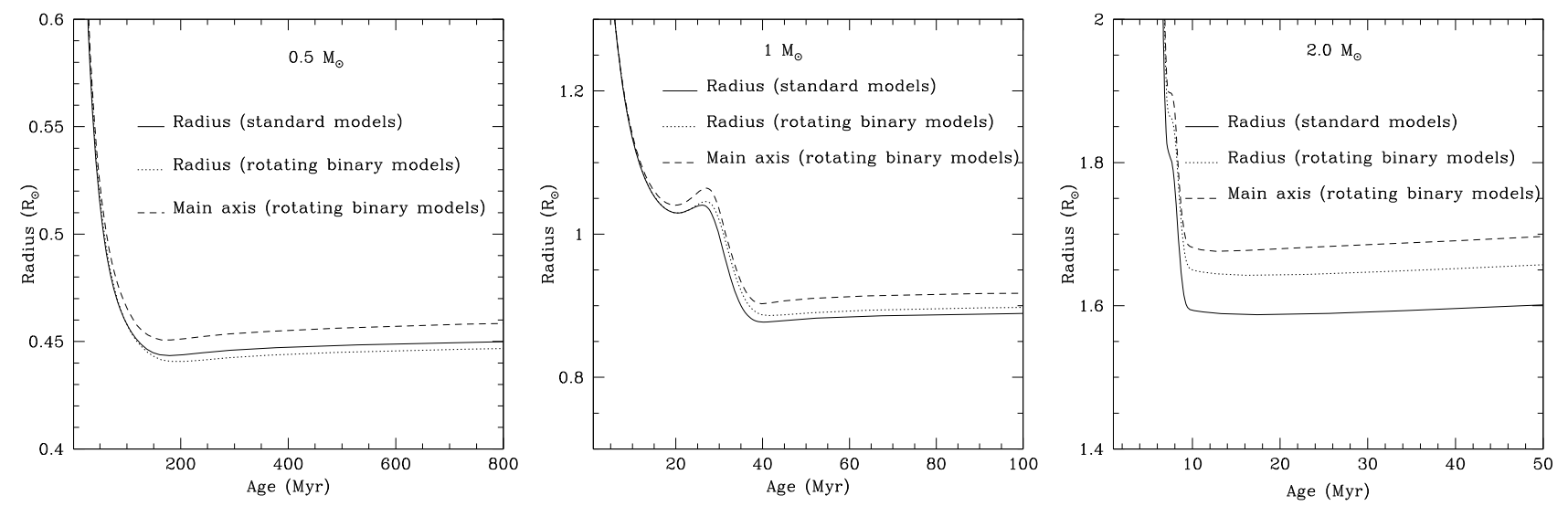

Fig. 6. Stellar radius and main axis of our standard models and rotating binary models. We report only the results for $0.5,1.0$ and $2.0 M_{\odot}$ models. The solid and dotted lines denote the radius of standard models and rotating binary models, respectively, while the dashed line stand for the radius along the main axis of the rotating binary models.

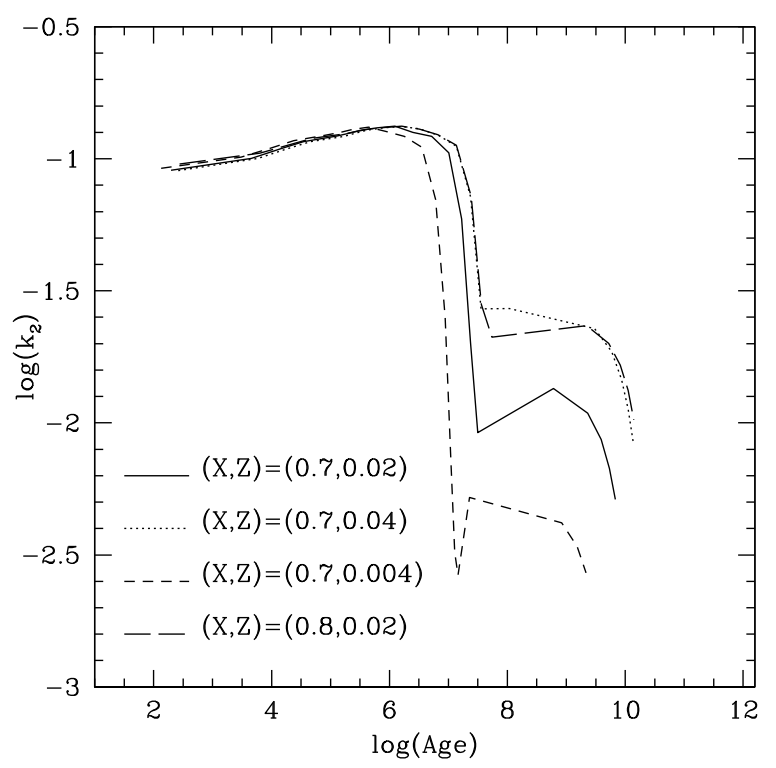

Fig. 7. $\log k_{2}$ as a function of the stellar age for a $1 M_{\odot}$ standard model, and for the initial chemical compositions $(X, Z)=(0.7,0.02)$, $(0.07,0.04),(0.7,0.004)$, and $(0.8,0.02)$.

for $\alpha=1.5$ and the initial chemical compositions above mentioned are shown, one can have an insight on how the metallicity affects the value of the second-order internal structure constant: during the first $1 \mathrm{Myr}$ the chemical composition does not alter $\log k_{2}$, but it becomes important from this age on; the metalpoorer stars evolve to more centrally condensed configurations (lower values of $\log k_{2}$ ) than their metal-richer counterparts. As it can also be seen from Fig. 7, using the initial chemical composition $(X, Z)=(0.7,0.04)$ (dotted lines) produces roughly the same effect on the evolution of $\log k_{2}$ as using the initial chemistry $(X, Z)=(0.8,0.02)$ (long-dashed lines).

In Table 4 we list the values at the ZAMS of the internal structure constants obtained from our standard and distorted $1 M_{\odot}$ models and also from other works in the literature. The values for our standard models were obtained with the same initial chemical compositions of Fig. 7; they can be compared with the values by, e.g., H87, CG89a and CG92.
Table 4. Values of $\log k_{2}$ for a $1 M_{\odot}$ star model at the ZAMS, obtained with our models (standard and distorted) and those previously published in the literature.

\begin{tabular}{lccc}
\hline \hline$(X, Z)$ & $\alpha_{\text {MLT }}$ & Reference & $\log \left(k_{2}\right)$ \\
\hline & 2.0 & H87 & -1.768 \\
$(0.7,0.02)$ & 2.0 & CG89a & -1.747 \\
& 2.0 & (standard) & -1.804 \\
& 1.5 & CG92 & -1.619 \\
& 1.5 & (standard) & -1.975 \\
$(0.7,0.04)$ & 2.0 & H87 & -1.614 \\
& 2.0 & (standard) & -1.548 \\
& 1.5 & (standard) & -1.628 \\
$(0.7,0.004)$ & 2.0 & H87 & -2.035 \\
& 2.0 & (standard) & -2.181 \\
& 1.5 & (standard) & -2.281 \\
$(0.8,0.02)$ & 2.0 & H87 & -1.578 \\
& 2.0 & (standard) & -1.688 \\
$(0.7,0.02)$ & 1.5 & (standard) & -1.776 \\
& 1.68 & C04 & -1.583 \\
& 1.68 & (standard) & -1.859 \\
$(0.748,0.004)$ & 1.68 & (distorted) & -1.895 \\
& 1.68 & C05 & -1.840 \\
& 1.68 & (standard) & -2.112 \\
$(0.73,0.010)$ & 1.68 & (distorted) & -2.145 \\
& 1.68 & C06b & -1.682 \\
& 1.68 & (standard) & -1.981 \\
& 1.68 & (distorted) & -2.004 \\
$(0.64,0.04)$ & 1.68 & C07 & -1.523 \\
& 1.68 & (standard) & -1.852 \\
& 1.68 & (distorted) & -1.866 \\
\hline & & &
\end{tabular}

We start by noting that for the initial chemical composition $(X, Z)=(0.7,0.02)$ and $\alpha=1.5$, our $1 M_{\odot}$ model produces a lower value of $\log k_{2}$ than that obtained by CG92, while for the same metallicity and $\alpha=2.0$, it produces a lower value of $\log k_{2}$ than those of CG89a and H87. Now, let us consider the $\alpha$ that fits the sun for each model, namely $\alpha=1.5$ for this work and CG92, and $\alpha=2.0$ for CG89a and H87. Still keeping the same metallicity, our models produce the most centrally condensed $1 M_{\odot}$ star, followed by that of H87, CG89a and CG92, in this order. These differences between our values of $\log k_{2}$ and those by CG89a and CG92 can be attributed to the use of different opacities, as they used Los Alamos Opacity Libraries and OPAL (Rogers \& Iglesias 1992) opacities respectively, while we used more recent OPAL opacities from Iglesias \& Rogers (1993); 
in addition, CG92 considered mass loss in their models. It is worth noting that although CG92 used more updated opacities than CG89a, for the case of $(X, Z)=(0.7,0.02)$ the former authors obtained a value of $k_{2}$ for their $1 M_{\odot}$ model at the ZAMS 1.34 times greater than that obtained by the latter ones. On the other hand, the CG92 models produce lower values of $k_{2}$ than those of CG89a for masses greater than $1.1 M_{\odot}$.

For the remaining initial chemical compositions, we can only compare our $1 M_{\odot}$ standard model with those by $\mathrm{H} 87$. For the case of $\alpha=2.0$, we notice that $\mathrm{H} 87$ obtained less mass-concentrated models for $(X, Z)=(0.7,0.004)$ and $(X, Z)=(0.8,0.02)$ than us, while for $(X, Z)=(0.7,0.04)$ it is the opposite. We can attribute this fact to two reasons: (1) although H87 obtained the lowest values of $k_{2}$ for one of the four metalicities used in this comparison, he used older opacities from Cox $\&$ Stewart (1969); and (2) our best fit to the sun is obtained with $\alpha=1.5$, not with $\alpha=2.0$. Regarding this last point, if we turn our attention to the models for which the value of $\alpha$ can reproduce the solar radius at the solar age ( $\alpha=1.5$ for this work and $\alpha=2.0$ for H87), we see that our models again produce a more centrally condensed star than those of H87, independent of the chemistry.

Finally, we compare the $\log \left(k_{2}\right)$ values from our distorted models with those of $\mathrm{C} 04, \mathrm{C} 05, \mathrm{C} 06 \mathrm{~b}$ and $\mathrm{C} 07$. In order to perform suitable comparisons, we computed additional $1 M_{\odot}$ models with the same physical inputs used in those works, namely $\alpha=1.68$ and initial chemical compositions of $(X, Z)=(0.7,0.02),(X, Z)=(0.748,0.004)$, $(X, Z)=(0.730,0.010)$, and $(X, Z)=(0.64,0.04)$; the corresponding values of $\log k_{2}$ at the ZAMS are shown in the lower part of Table 4. We note that although the effects of tides and rotation are also considered in the models by Claret and collaborators (Claret 1998; Claret 2008, personal communication), their values presented in Table 4 correspond to isolated, non-rotating stars; for comparison purposes, we also present in it our values calculated for isolated, non-rotating stars with the same masses and chemical composition as well as the corresponding ones including tidal and rotational distortions. For all chemical compositions, our models again produce more mass-concentrated stars than those by C04, C05, C06b, and C07. For the mass range of this comparison, these differences in the $\log k_{2}$ values can be due to the presence of distortion effects in our models and also to the use of a different equation of state: while we use OPAL (Rogers et al. 1996) and Mihalas et al. (1988) EOS, those authors use the CEFF (Christensen-Dalsgaard \& Däppen 1992) EOS.

Figure 8 presents a graphical comparison between the values of $\log k_{2}$ obtained in this work and those obtained by $\mathrm{H} 87$, CG89a, CG92, C04, C05, C06b, and C07. This figure gives an easier overview of Table 4.

\section{Comparison between theory and observations}

Double-lined eclipsing binary systems are good candidates to test evolutionary models, but very few systems are as adequate as EK Cep $(P=4.42)$. The mass and radius of its primary component are $M_{1}=2.029 \pm 0.023 M_{\odot}$ and $R_{1}=1.579 \pm$ $0.007 R_{\odot}$, while, for the secondary, $M_{2}=1.124 \pm 0.012 M_{\odot}$ and $R_{2}=1.315 \pm 0.006 R_{\odot}$ (Claret 2006a). As mentioned by Claret (2006a), EK Cep has accurate determination of absolute parameters, at least concerning masses and radii (see Table 5); its secondary component is a pre-MS star, the apsidal motion presented by the system has a high relativistic contribution, the less massive component has its Lithium abundance determined observationally, the metallicity of the binary is evaluated, and,

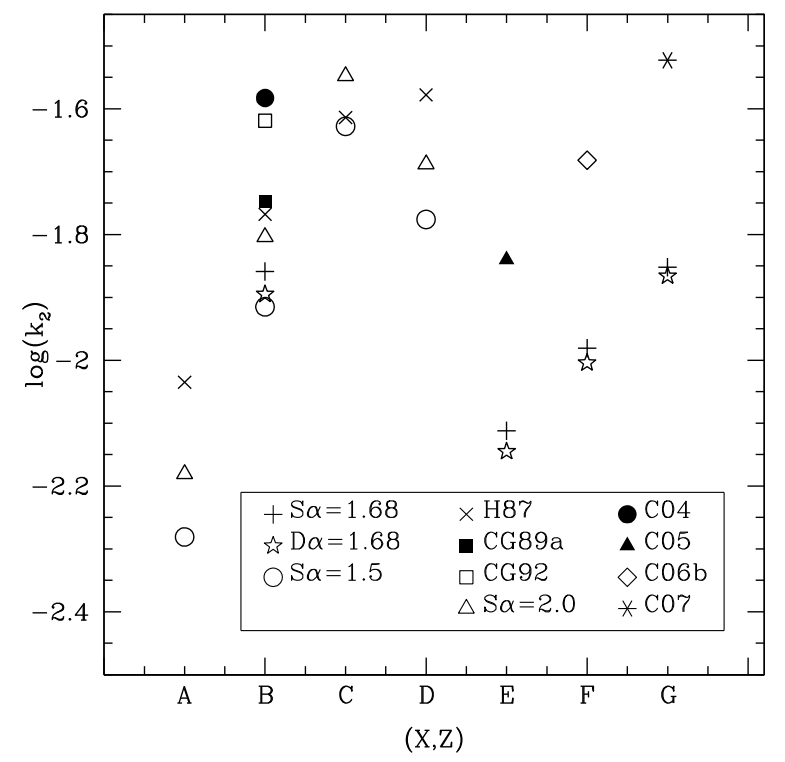

Fig. 8. A schematic comparison between $\log k_{2}$ values obtained with our standard models ( $\mathrm{S} \alpha=1.5$ and $\mathrm{S} \alpha=2.0$ ), our distorted models $(\mathrm{D} \alpha=1.68)$ and those obtained by other authors. The values plotted are the same as in Table 4, and the corresponding initial chemical compositions are the following, labeled A to $\mathrm{G}$ respectively: $(X, Z)=(0.7,0.004),(0.7,0.02),(0.7,0.04),(0.8,0.02),(0.748,0.004)$, $(0.73,0.01)$, and $(0.64,0.04)$.

Table 5. Absolute dimensions of EK Cep (Claret 2006a; Tomkin 1983).

\begin{tabular}{lrr}
\hline \hline Parameter & Primary & Secondary \\
\hline Mass $\left(M_{\odot}\right)$ & $2.029 \pm 0.023$ & $1.124 \pm 0.012$ \\
Radius $\left(R_{\odot}\right)$ & $1.579 \pm 0.007$ & $1.315 \pm 0.006$ \\
$\log (g)(\mathrm{cgs})$ & $4.349 \pm 0.010$ & $4.251 \pm 0.006$ \\
$\log \left(L / L_{\odot}\right)$ & $1.17 \pm 0.04$ & $0.19 \pm 0.07$ \\
$\log \left(T_{\text {eff }}\right)(\mathrm{K})$ & $3.954 \pm 0.010$ & $3.756 \pm 0.015$ \\
$v_{\text {rot }}\left(\mathrm{km} \mathrm{s}^{-1}\right)$ & $23 \pm 2$ & $10.5 \pm 2$ \\
\hline
\end{tabular}

further, the rotational velocity of each component is observationally measured. Besides that, EK Cep is the only known pre-MS system with measured apsidal motion. For all these reasons, we choose EK Cep to test our new models.

EK Cep was discovered as an eclipsing binary by Strohmeier (1959) from photographic observations. Photometric elements and a spectroscopic orbit of the system were first presented by Ebbighausen (1966a,b), and further revised by other authors. The apsidal motion of EK Cep was first reported in Khaliullin (1983a). Tomkin (1983) determined the masses and the radii for its primary and secondary components. He also noted that the secondary is oversized in comparison with a main-sequence star with the same mass and supposed that it might still be contracting towards the main sequence. Hill \& Ebbighausen (1984) concluded that both components are zero-age main-sequence objects, despite Tomkin (1983) suggestion about the secondary. Giménez (1985) obtained a good agreement between theoretical and observationally determined apsidal motion rates. Popper (1987) reported some anomalies in the secondary of EK Cep, such as low effective gravity and temperature as well as the excess radiation in the blue band, that appear to be consistent with the hypothesis of the pre-MS nature of this star. From high-resolution spectroscopy in the LiI $\lambda 6708 \AA$ region, Martín \& Rebolo (1993) determined the lithium abundance of EK Cep 
$\mathrm{B}$ and provided new evidence that it has not settled onto the ZAMS. Claret et al. (1995b) compared the observed parameters of EK Cep with theoretically predicted values; they derived a common age for the system around $2 \times 10^{7} \mathrm{yr}$ and confirmed the fainter component as a pre-MS star, while the more massive companion is in the beginning of the Hydrogen-burning phase. They estimated a Newtonian apsidal motion rate that is in agreement with the observations, considering a predicted relativistic contribution of about $40 \%$. Those authors also found that the lithium depletion computed by their models is consistent with the abundances determined by Martín \& Rebolo (1993).

The evolutionary status of EK Cep was studied by other authors. Yildiz (2003) modeled the component stars by invoking a rapidly rotating core for the primary. Marques et al. (2004) investigated the role of overshooting on the modeling of pre-MS evolution of the secondary. Claret (2006a) presented the most recent analysis about EK Cep; due to problems with the empirical determination of the effective temperatures of the component stars, he adopted the effective temperature ratio $\left(\mathrm{TR}=T_{\mathrm{eff}, 2} / T_{\mathrm{eff}, 1}\right)$, which is better determined from the light curve analysis than their absolute values. Inconsistencies found in the photometric distances for both components support this approach. Claret (2006a) used other constraints in his analysis as the radii, apsidal motion and lithium depletion. With a rotating model (assuming local conservation of the angular momentum), $\alpha=1.4$ and $(X, Z)=(0.7075,0.0175)$, he fitted the radii and TR in the same isochrone $\left(24.2 \times 10^{6} \mathrm{yr}\right)$.

As emphasized by Claret (2006a), before computing stellar models for a given star it is fundamental to define clearly which observational parameters will be used as constraints. Following the same approach as Claret (2006a), we adopt the masses, radii and TR, leaving the apsidal motion, rotational velocities and lithium depletion to be used as additional constraints after obtaining an acceptable solution. We then computed rotating binary models with metallicities as close as possible to the solar metallicity, following the conclusion by Martín \& Rebolo (1993) that the EK Cep secondary has a metal contents typical of an young disk, solar-type star. Using an initial chemical composition of $(X, Z)=(0.67,0.017)$ and the mixing length parameter $\alpha=1.5$, we obtained a model that reproduces the radii and TR for EK Cep within the uncertainties, as shown in Figs. 9 and 10, deriving two possible age intervals for the system, $15.5-16.7 \times 10^{6}$ and $18.9-19.3 \times 10^{6} \mathrm{yr}$. The input values we used are different from those by Claret (2006a) since we are using different stellar models, with different physics. By using his inputs, i.e. $(X, Z)=(0.07075,0.0175)$ and $\alpha=1.4$, we were not able to reproduce, simultaneously, both stellar radii at the same age interval; this is due to the fact that each model needs slightly different inputs for adjusting to a same set of observed data.

According to our radii and TR analysis, the two derived age intervals allow a reasonable adjustment of the observational data; we used additional data (rotational velocities of each component, lithium depletion and apsidal motion constants) to constrain even more the system's age estimate.

We first tried to reproduce the observed rotational velocites of EK Cep primary and secondary components at at least one of the derived age intervals; in order to do so, we needed to use an initial angular momentum for each component lower than that prescribed by Eq. (72). In Fig. 11 we show the time evolution of the rotational velocities for EK Cep determined masses; the vertical lines correspond to the two age intervals that fit simultaneously the stellar radii and temperature ratio, while the horizontal ones delimit the observed EK Cep rotational velocities.

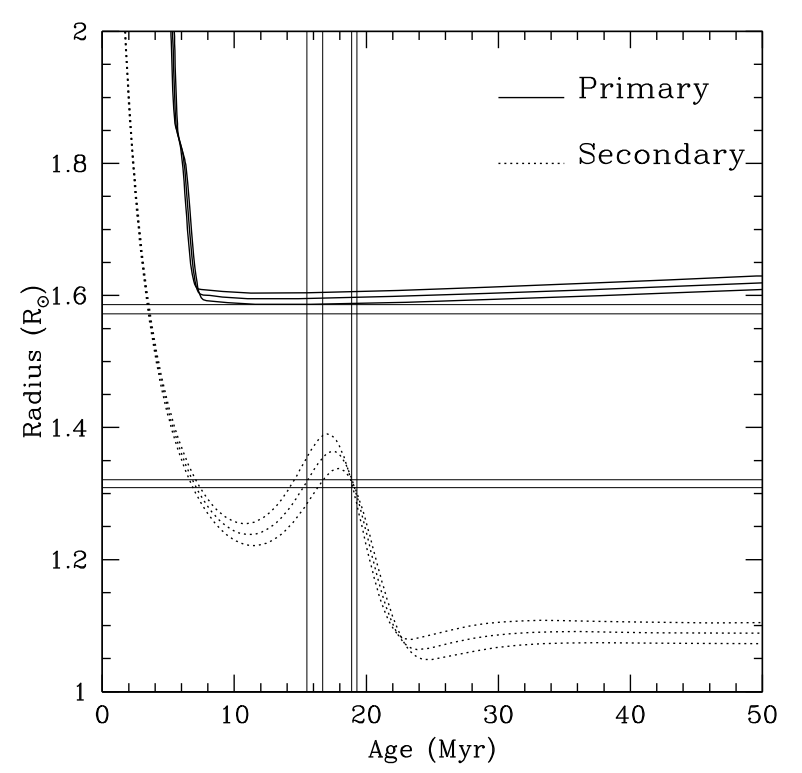

Fig. 9. Radii predicted by our binary rotating models for EK Cep components determined masses. The additional tracks refer to the maximum and minimum mass of each component, according to their errors. Full lines denote the primary while dotted ones denote the secondary. Note that there is an acceptable agreement between radii and effective temperature ratio (see Fig. 10) for two age intervals (vertical lines). Horizontal lines represent the error bars in radii determinations.

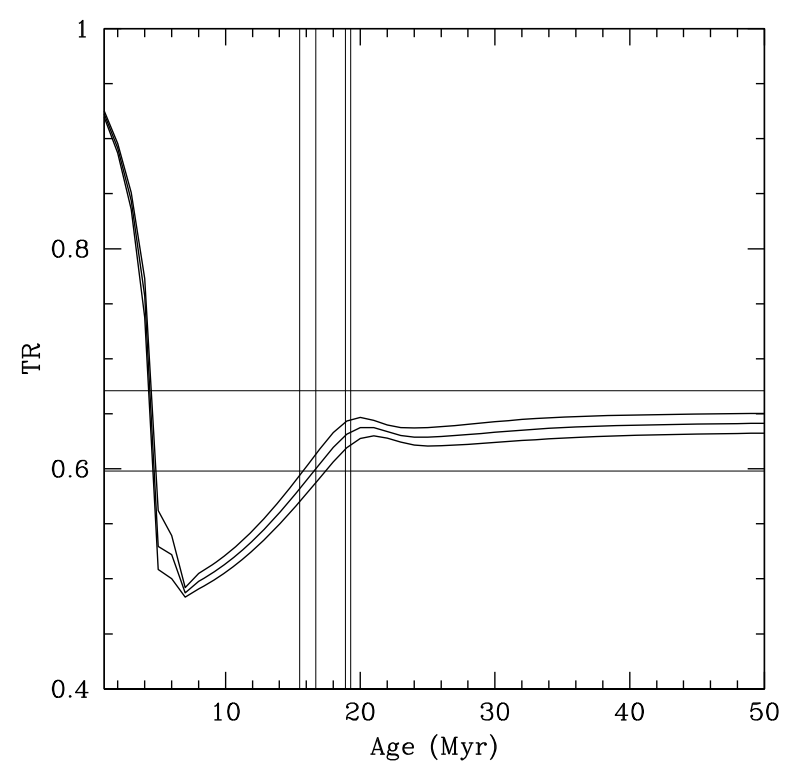

Fig. 10. Effective temperature ratio $\left(\mathrm{TR}=T_{\mathrm{eff}, 2} / T_{\mathrm{eff}, 1}\right)$ predicted by our binary rotating models for EK Cep components determined mass. The additional tracks refer to the maximum and minimum mass of each component star, according to the errors in their determination. The vertical lines correspond to the same age intervals as in Fig. 9. Horizontal lines represent the error bars in TR determinations.

From that figure, one can see that the older age interval does not match the observed rotational velocity of the secondary star.

The lithium depletion of our rotating binary models is shown in Fig. 12, in which the Li contents is plotted against the stellar age. We started from an initial lithium abundance of $\log (\mathrm{Li} / \mathrm{H})=3.1$ (as D’Antona \& Montalbán 2003). As 


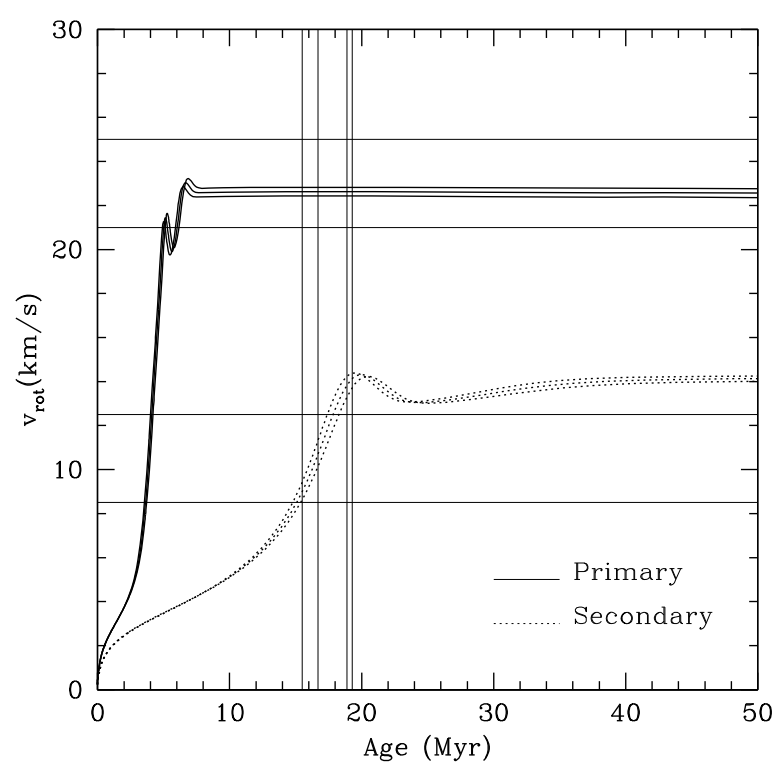

Fig. 11. Time evolution of rotational velocities along the pre-MS for EK Cep determined masses. Horizontal lines represent the error bars in rotational velocity determinations. Curve triplets and vertical lines defined as in Fig. 9.

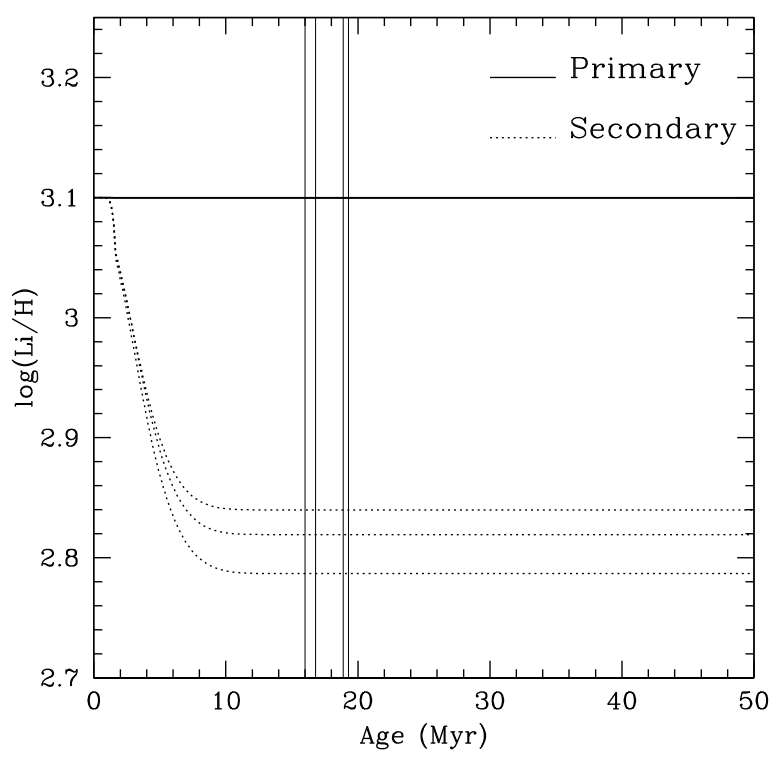

Fig. 12. Time evolution of lithium abundances for EK Cep determined masses according to our rotating binary models. Curve triplets and vertical lines defined as in Fig. 9. Note that the curves corresponding to the primary mass and to its error bar collapse to a single horizontal line.

expected, we do not find any depletion for the primary during its pre-MS evolution due to its higher mass; for the secondary, we find a depletion of about $0.28 \mathrm{dex}$ in both possible age intervals for EK Cep. This corresponds to a lithium abundance of $\log (\mathrm{Li} / \mathrm{H})=2.82 \pm 0.03$, consistent with the surface value of $\log (\mathrm{Li} / \mathrm{H})=3.1 \pm 0.3$ measured by Martín \& Rebolo (1993). The lithium depletion does not allow us to choose between the two possible age intervals for the system, since it does not vary in the whole interval from $10 \mathrm{Myr}$ to $50 \mathrm{Myr}$.

Finally, we investigated the apsidal motion rate of EK Cep with the same rotating binary models. The values for the

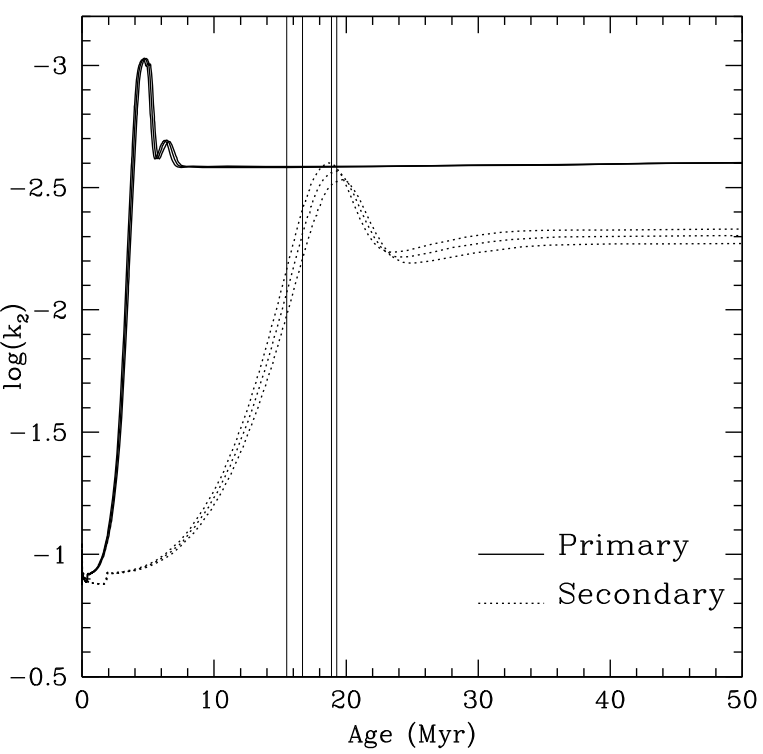

Fig. 13. The second-order apsidal motion constant as a function of the stellar age for EK Cep, as obtained from our rotating binary models. Curve triplets and vertical lines defined as in Fig. 9.

Table 6. Summary of the quantities related to the apsidal motion.

\begin{tabular}{lrrr}
\hline \hline & $\log k_{2}$ & $U$ (years) & $\dot{\omega}$ (o/cycle) \\
\hline Our models (AI1) & -2.3 & 5800 & 0.00076 \\
& \pm 2 & \pm 800 & \pm 12 \\
Our models (AI2) & -2.57 & 7070 & 0.00061 \\
& \pm 2 & \pm 50 & \pm 1 \\
Observed & -2.09 & 4500 & 0.00097 \\
& \pm 9 & \pm 700 & \pm 15 \\
Claret (2006a) & -2.116 & 4600 & 0.00095 \\
& \pm 6 & \pm 400 & \pm 8 \\
\hline
\end{tabular}

orbital eccentricity $(0.109 \pm 0.003$ degrees $)$ and the orbital inclination $(89.3 \pm 0.1$ degrees) were taken from Petrova \& Orlov (1999). The anomalistic period of EK Cep is $P_{\text {an }}=4.4278062 \pm$ 0.0000005 days (Claret 2006a). In Fig. 13 we show the variation of $\log \left(k_{2}\right)$ for the observed masses (with their errors) as a function of age. As before, the vertical lines indicate the age intervals that fit both the radii and effective temperature ratio of EK Cep components, and from which we took the values of the internal structure constants to derive the apsidal motion rate.

For the first age interval, 15.5-16.7 Myr (AI1), our models result in $\mathrm{k}_{2}$ values of $0.00261 \pm 0.00001$ and $0.007 \pm 0.003$ for the primary and the secondary components, respectively. From Eq. (10) we obtain $\log \bar{k}_{2 \text { theo }}=-2.3 \pm 0.2$ as the mean value of the second-order apsidal motion constant of the system. The Newtonian contribution of the apsidal motion rate is obtained from Eqs. (3) and (10), resulting in $\dot{\omega}_{\mathrm{N}}=(0.00033 \pm 0.00011) \%$ cycle. We also calculated the relativistic contribution of the advance of periastron, $\dot{\omega}_{\mathrm{R}}=(0.000434 \pm 0.000005)^{\circ} /$ cycle, which, based on our models, corresponds to about $49-68 \%$ of the total rate. From these values, our models result in an apsidal motion rate of $\dot{\omega}=(0.00076 \pm 0.00012) \%$ cycle and, consequently, an apsidal period of $U_{\text {theo }}=(5800 \pm 800) \mathrm{yr}$; these values are comparable with the observed ones. However, for the second age interval, 18.9-19.3 Myr (AI2), we were not able to reproduce the observed apsidal motion of EK Cep by using our rotating binary models. As can be seen in Table 6, the apsidal motion quantities 
we found in AI2 (log $k_{2}$, apsidal period $U$ and apsidal motion rate $\dot{\omega}$ ) are not in agreement with the observed values, even considering the error bars.

Claret (2006a) used results of a series of works (Khaliullin 1983b; Giménez \& Margrave 1985; Hill \& Ebbighausen 1984; Claret et al. 1995b, and others) to derive the observed apsidal motion rate of this system. He reported the observed mean value of the internal structure constant as $\log \bar{k}_{2}=-2.09 \pm 0.09$, which is equivalent to an observed apsidal motion of about $\dot{\omega}_{\text {obs }}=(0.00097 \pm 0.00015)^{\circ} /$ cycle, after applying the relativistic correction. This apsidal motion rate produces an apsidal period of $U_{\text {obs }}=(4500 \pm 700)$ years. From Table 6 , where our results, those by Claret (2006a) and the observed determinations are summarized, it can be seen that our predicted values for AI1 are in good agreement with the observed ones (as well as those by Claret 2006a), and the differences lie within the errors.

At first glance, we could be tempted to conclude that the second age interval AI2 gives a better agreement for the EK Cep system than the first one due to the following reasons: (1) at AI2, the radius of the secondary component constrains the age of the system to a much narrower age interval, as shown in Fig. 9; and (2) the TR curves produced by our models lie very well inside the AI2 age interval, but are fitted just marginally for the earlier AI1 age interval (Fig. 10). However, in that age interval our rotating binary models were not able to reproduce neither the rotational velocity of the secondary star (Fig. 11) nor the apsidal motion rate for the system (Table 6), while the opposite did happen for AI1. Therefore, AI1 seems to be more suitable than AI2 as the age interval for investigating the evolutionary status of EK Cep. For these reasons, with regard to our models, we suggest that the age of EK Cep is between 15.5 and 16.7 Myr.

\section{Conclusions}

We computed stellar evolutionary models that take into account the combined effects of rotation and of tidal forces due to a companion star, obtaining values of the internal structure constants for low-mass, pre-MS stars. Our approximation for binary model calculations (with effects of tidal forces) do not include the pseudo-potential centrifugal terms arising from the orbital motion of the components around the center of mass of the system. For all sets of models, namely standard, binary, rotating and rotating binary models, we tabulated the internal structure constants and the gyration radii for ZAMS models and their time evolution. Distorted models result in more mass-concentrated stars and produce larger gyration radii at the ZAMS than standard ones.

The non-standard evolutionary tracks are cooler than their standard counterparts, mainly for low-mass stars. Regarding the internal structure of our stellar models, we verified that tidal effects act in the same way as rotational ones but in a smaller scale; the relative importance of these effects on the apsidal motion constants depend mainly on the orbital separation and the star's rotation rate. Besides, rotationally and tidally distorted models produce some effects in the physical quantities of a star, in comparison with the standard models, that are in one direction, for masses below $\sim 0.7 M_{\odot}$, and in the opposite direction, for masses above this threshold. Though Sackmann (1970) associated this behavior in distortion effects with the transition from the $\mathrm{p}-\mathrm{p}$ chain to the $\mathrm{CNO}$ cycle, this transition occurs in a mass range about $1.3-1.5 M_{\odot}$, considerably above the threshold we observed with our models for the change of behavior of the physical quantities of the stars.
We also found that, for masses lower than $0.5 M_{\odot}$, the relative importance of the second-order internal structure constants over those of higher order is much lower than for the mass interval of 1.5-3.8 $M_{\odot}$; hence, in the low-mass range, the usual assumption that the harmonics of order greater than $j=2$ can be neglected seems not to be entirely justified except maybe for computing the apsidal motion rate $\dot{\omega}$.

Our results on internal structure constants were compared with those available in the literature and found to be compatible with them. The $k_{2}$ values obtained from our standard models are smaller than those last published by H87, CG89a and CG92, except for a given model by $\mathrm{H} 87$ with the inputs $(X, Z)=(0.7,0.04)$ and $\alpha=2.0$. Our rotating binary models produce internal structure constants even smaller, resulting in more mass-concentrated configurations than the models by $\mathrm{C} 04, \mathrm{C} 05, \mathrm{C} 06$, and $\mathrm{C} 07$. These comparisons were made for representative $1 M_{\odot}$ models, with the same initial chemical compositions of those works.

Using our set of evolutionary tracks for rotating binary models, we also investigated the evolutionary status of the interesting double-lined eclipsing binary system EK Cep. Its primary, a $2.029 M_{\odot}$ star, seems to be in the hydrogen-burning phase, and its secondary, a solar-like star $\left(1.124 M_{\odot}\right)$, is confirmed as a pre-MS star. By using a model with an initial chemical composition of $(X, Z)=(0.67,0.017)$ and a mixing length parameter of $\alpha=1.5$, we reproduced stellar radii and the effective temperature ratio of EK Cep in two different age intervals. We also followed the lithium contents during the pre-MS evolution of both components; as expected, we do not find any significant depletion for the primary, while the Li depletion for the secondary agrees with the observed values within the uncertainties for both age intervals. However, for the later age interval of 18.9-19.3 Myr, we were not able to fit neither the observed rotational velocity of both components nor the observed apsidal motion rate of the system. On the other hand, all observed quantities used in our analysis were reproduced in the earlier age interval of 15.5-16.7 Myr. Although being a broader range for the age of EK Cep and introducing greater error bars to the apsidal motion quantities, it seems to give a more confident age for the system.

In this paper, we presented a first attempt to introduce the combined structural effects of tides and rotation in the ATON evolutionary code. Even with simple assumptions about the secondary component of a binary system, we could verify the importance of those combined effects on stellar structure and evolution. Work is in progress, where we remove the assumption that the disturbing star can be treated as a point mass, include the pseudo-potential centrifugal terms caused by the orbital motion and consider the simultaneous evolution of the two components of a binary system.

Acknowledgements. The authors thank Drs. Francesca D'Antona (INAF-OAR, Italy) and Italo Mazzitelli (INAF-IASF, Italy) for granting them full access to the ATON evolutionary code. We also are grateful to the referee, Dr. B. Willems, for his many comments and suggestions that helped to improve this work. The financial support from the Brazilian agencies CAPES, CNPq and FAPEMIG is also acknowledged.

\section{References}

Alexander, D. R., \& Ferguson, J. W. 1994, ApJ, 437, 879

Batten, A. H. 1973, in Binary and Multiple Systems of Stars (Oxford: Pergamon Press)

Böhm-Vitense, E. 1958, Z. Astroph., 46, 108

Brooker, R. A., \& Olle, T. W. 1955, MNRAS, 115, 101

Canuto, V. M., Goldman, I., \& Mazzitelli, I. 1996, ApJ, 473, 550 
Carson, T. R. 1976, ARA\&A, 14, 95

Caughlan, G. R., \& Fowler, W. A. 1988, Atomic Data Nucl. Tab., 40, 283 Chaboyer, B., Demarque, P., \& Pinsonneault, M. H. 1995, ApJ, 441, 865 Chandrasekhar, S. 1933, MNRAS, 93, 390

Christensen-Dalsgaard, J., \& Däppen, W. 1992, A\&AR, 4, 267

Claret, A. 1995, A\&AS, 109, 441

Claret, A. 1998, A\&AS, 131, 195

Claret, A. 1999, A\&A, 350, 56

Claret, A. 2004, A\&A, 424, 919 (C04)

Claret, A. 2005, A\&A, 440, 647 (C05)

Claret, A. 2006a, A\&A, 445, 1061

Claret, A. 2006b, A\&A, 453, 769 (C06b)

Claret, A. 2007, A\&A, 467, 1389 (C07)

Claret, A., \& Giménez, A. 1989a, A\&AS, 81, 1 (CG89a)

Claret, A., \& Giménez, A. 1989b, A\&AS, 81, 37

Claret, A., \& Giménez, A. 1991, A\&AS, 87, 507

Claret, A., \& Giménez, A. 1992, A\&AS, 96, 255 (CG92)

Claret, A., \& Giménez, A. 1993, A\&A, 277, 487

Claret, A., \& Giménez, A. 2001, LNP, 563, 1

Claret, A., \& Willems, B. 2002, A\&A, 388, 518

Claret, A., Giménez, A., \& Martín, E. L. 1995, A\&A, 302, 741

Cox, A. N., \& Stewart, J. N. 1969, Nautshnij Informatsij, 15, 1

Cowling, T. G. 1938, MNRAS, 98, 734

D’Antona, F., \& Montalbán, J. 2003, A\&A, 412, 213

Ebbighausen, E. G. 1966a, AJ, 71, 642

Ebbighausen, E. G. 1966b, AJ, 71, 730

Endal, A. S., \& Sofia, S. 1976, ApJ, 210, 184

Fliegner, J., \& Langer, N. 1995, in Wolf-RAyet Stars: Binaries, Colliding Winds, Evolution, ed. K. A. van der Hucht, \& P. M. Willians (Kluwer), IAU Symp., 163

Giménez, A. 1985, ApJ, 297, 405

Giménez, A., \& Margrave, T. E. 1985, AJ, 90, 358

Hadjidemetriou, J. 1967, Adv. Astr. Astrophys., 5, 131

Hejlesen, P. M. 1987, A\&AS, 69, 251 (H87)

Heger, A., Langer, N., \& Woosley, S. E. 2000, ApJ, 528, 368

Hilditch, R. W. 2001, in An Introduction to Close Binary Stars (Cambridge University Press)

Hill, G., \& Ebbighausen, E. G. 1984, AJ, 89, 1256

Iglesias, C. A., \& Rogers, F. J. 1993, ApJ, 412, 752

Jeffery, C. M. 1984, MNRAS, 207, 323

Khaliullin, Kh. F. 1983a, AZh., 60, 72

Khaliullin, Kh. F. 1983b, Sov. Astron., 27, 43

Kawaler, S. D. 1987, PASP, 99, 1322

Keller, G., \& Meyerott, R. E. 1955, ApJ, 122, 32

Kippenhahn, R., \& Thomas, H.-C. 1970, in Stellar Rotation, ed. A. Slettebak (Dordrecht: Reidel) (KT70)

Kippenhahn, R., \& Weigert, A. 1994, Stellar Structure and Evolution (SpringerVerlag)

Kopal, Z. 1959, in Close Binary Systems, (New York: Wiley)

Kopal, Z. 1960, in Figures of Equilibrium of Celestial Bodies (The University of Wisconsin Press)

Kopal, Z. 1972, Adv. Astron. Astrophys., 9, 1

Kopal, Z. 1974, Ap\&SS, 27, 389

Kopal, Z. 1978, in Dynamics of Close Binary Systems (Dordrecht: Reidel)

Kopal, Z. 1989, in The Roche Problem, (Dordrecht: Kluwer Academic Publishers)

Kushawa, R. S. 1957, ApJ, 125, 242
Landin, N. R., Ventura, P., D’Antona, F., Mendes, L. T. S., \& Vaz, L. P. R. 2006, A\&A, 456, 269

Law, W.-Y. 1980, Ph.D. Thesis, Yale University

Levi-Civita, T. 1937, Amer. J. Math., 59, 225

Marques, J. P., Fernandes, J., \& Monteiro, M. J. P. F. G. 2004, A\&A, 422, 239

Martín, E. L., \& Rebolo, R. 1993, A\&A, 274, 274

Martín, E. L., \& Claret, A. 1996, A\&A, 306, 408

Martynov, D. Ya. 1948, Izv. Engelhardt Obs., 25

Martynov, D. Ya. 1973, in Eclipsing Variable Stars, ed. V. P. Tsesevich, IPST Astrophys. Library, Jerusalem

Mendes, L. T. S. 1999, Ph.D. Thesis, Federal University of Minas Gerais

Mendes, L. T. S., D’Antona, F., \& Mazzitelli, I. 1999, A\&A, 341, 174

Meynet, G., \& Maeder, A. 1997, A\&A, 321, 465

Meynet, G., \& Maeder, A. 2000, A\&A, 361, 101

Maeder, A., \& Meynet, G. 2000, A\&A, 361, 159

Maeder, A., \& Meynet, G. 2001, A\&A, 373, 555

Meynet, G., \& Maeder, A. 2002, A\&A, 390, 561

Maeder, A., \& Zahn, J.-P. 1998, A\&A, 334, 1000

Mihalas, D., Dappen, W., \& Hummer, D. G. 1988, ApJ, 331, 815 (M88)

Mohan, C., Saxena, R. M., \& Agarwal, S. R. 1990, Ap\&SS, 163, 23

Motz, L. 1952, AJ, 115, 562

Petrova, A. V., \& Orlov, V. V. 1999, AJ, 117, 587

Pinsonneault, M. H. 1988, Ph.D. Thesis, Yale University

Pinsonneault, M. H., Kawaler, S. D., \& Demarque, P. 1990, ApJS, 74, 501

Popper, D. M. 1987, ApJ, 313, 81

Press, W. H., Teukolsky, S. A., Vetterling, W. T., \& Flannery, B. P. 1992, Numerical Recipes in Fortran 77: The art of Scientific Computing (Cambridge University Press)

Rogers, F. J., \& Iglesias, C. A. 1992, ApJS, 79, 507

Rogers, F. J., Swenson, F. J., \& Iglesias, C. A. 1996, ApJ, 456, 902

Ruciński, S. M. 1969, Acta Astron., 19, 125

Ruciński, S. M. 1988, AJ, 95, 1895

Russell, H. N. 1928, MNRAS, 88, 642

Sackmann, I. J. 1970, A\&A, 8, 76

Sackmann, I. J., \& Anand, S. P. S. 1969, ApJ, 155, 257

Sahade, J., \& Wood, F. B. 1978, in Interacting Binary Stars (Oxford: Pergamom Press)

Savonije, G. J., \& Papaloizou, J. C. B. 1983, MNRAS, 203, 581

Savonije, G. J., \& Witte, M. G. 2002, A\&A, 386, 211

Sterne, T. E. 1939, MNRAS, 99, 662

Strohmeier, W. 1959, kleine Veröff. Bamberg, 27

Schwarzschild, M. 1958, in Structure and Evolution of the Stars (Princeton Univ. Press)

Tomkin, J. 1983, ApJ, 271, 717

Ureche, V. 1976, ed. P. Eggleton (Dordrecht: Reidel), IAU Symp., 73

Ventura, P., Zeppieri, A., Mazzitelli, I., \& D’Antona, F. 1998, A\&A, 334, 953

Yildiz, M. 2003, A\&A, 409, 689

Willems, B., \& Claret, A. 2003, A\&A, 410, 289

Willems, B., van Hoolst, T., \& Smeyers, P. 2003, A\&A, 397, 973

Witte, M. G., \& Savonije, G. J. 1999a, A\&A, 341, 842

Witte, M. G., \& Savonije, G. J. 1999b, A\&A, 350, 129

Witte, M. G., \& Savonije, G. J. 2001, A\&A, 366, 840

Witte, M. G., \& Savonije, G. J. 2002, A\&A, 386, 222

Zahn, J.-P. 1966, AnAp., 29, 489

Zahn, J.-P. 1975, A\&A, 41, 329

Zahn, J.-P. 1977, A\&A, 57, 383

Zahn, J.-P. 1989, A\&A, 220, 112 